\title{
Nucleons in Two-Flavor Partially-Quenched Chiral Perturbation Theory
}

\author{
Silas R. Beane and Martin J. Savage \\ Department of Physics, University of Washington, \\ Seattle, WA 98195.
}

\begin{abstract}
Properties of the proton and the neutron are explored in partiallyquenched chiral perturbation theory with two non-degenerate light flavors. Masses, magnetic moments, matrix elements of isovector twist-2 operators and axial-vector currents are computed at the one-loop level in the chiral expansion.
\end{abstract}




\section{INTRODUCTION}

At some point in time lattice calculations will be able to determine the properties and interactions of the low-lying hadrons directly from QCD. Impressive progress is being made toward this goal, however present computational limitations necessitate the use of quark masses that are significantly larger than those of nature. Typically one has $m_{\pi}^{\text {latt. }} \sim 500 \mathrm{MeV}$. In order to make a connection between lattice calculations of the foreseeable future and nature, an extrapolation in the quark masses is required. Chiral perturbation theory $(\chi \mathrm{PT})$ provides a systematic description of low-energy QCD near the chiral limit, and this technique has been extended to describe both quenched QCD (QQCD) [1 5 and partially-quenched QCD (PQQCD) [6,7]. The hope is that future lattice simulations can be performed with quark masses that are small enough to guarantee a convergent chiral expansion, thus allowing a meaningful extrapolation to the quark masses of nature.

Recently, it has been shown how to include the low-lying octet and decuplet of baryons [8] into partially-quenched chiral perturbation theory $(\mathrm{PQ} \chi \mathrm{PT})$ [6,7]. As the pion mass presently obtainable in lattice simulations is not much smaller than the physical kaon mass, it is natural to consider a theory with three light valence-quarks and three light sea-quarks. The masses, magnetic moments and matrix elements of isovector twist-2 operators were computed at the one-loop level in $S U(6 \mid 3)_{L} \otimes S U(6 \mid 3)_{R}$ PQ $\chi$ PT [8]. The strange quark mass was taken to be different from the other two degenerate light quarks (isospin limit) and one of the sea-quarks was taken to have a mass different from the other two. However, many lattice simulations are performed with just two light quarks. In this work we determine several observables of the proton and the neutron at one-loop level in PQ $\chi \mathrm{PT}$ with two non-degenerate light quarks using $S U(4 \mid 2)_{L} \otimes S U(4 \mid 2)_{R} \mathrm{PQ} \chi \mathrm{PT}$. Isospin violation of electromagnetic origin is not considered.

In Section [1] we develop the general formalism for including baryons in $S U(4 \mid 2)_{L} \otimes$ $S U(4 \mid 2)_{R}$ PQ $\chi \mathrm{PT}$. The masses and magnetic moments of the proton and neutron are computed to one-loop order in the chiral expansion in Section III and IV, respectively. In Section $\mathrm{V}$ and VI we present one-loop level expressions for matrix elements of isovector twist-2 operators and axial-vector currents, respectively. We conclude in Section VII.

\section{II. $\mathbf{P Q} \chi \mathbf{P T}$}

The Lagrange density describing the quark-sector of PQQCD is

$$
\begin{aligned}
\mathcal{L} & =\sum_{a, b=u, d} \bar{q}_{V}^{a}\left[i \not D-m_{q}\right]_{a}^{b} q_{V b}+\sum_{\tilde{a}, \tilde{b}=\tilde{u}, \tilde{d}} \overline{\tilde{q}}^{\tilde{a}}\left[i \not D-m_{\tilde{q}}\right]_{\tilde{a}}^{\tilde{b}} \tilde{q}_{\tilde{b}}+\sum_{a, b=j, l} \bar{q}_{\text {sea }}^{a}\left[i \not D-m_{\text {sea }}\right]_{a}^{b} q_{\text {sea }, b} \\
& =\sum_{k, n=u, d, \tilde{u}, \tilde{d}, j, l} \bar{Q}^{k}\left[i \not D-m_{Q}\right]_{k}^{n} Q_{n}
\end{aligned}
$$

where the $q_{V}$ are the two light-quarks, $u$ and $d$, the $\tilde{q}$ are two light bosonic (ghost) quarks $\tilde{u}$ and $\tilde{d}$, and the $q_{\text {sea }}$ are the two sea-quarks $j$ and $l$. The left- and right-handed valence-, sea-, and ghost-quarks are combined into column vectors

$$
Q_{L}=(u, d, j, l, \tilde{u}, \tilde{d})_{L}^{T}, Q_{R}=(u, d, j, l, \tilde{u}, \tilde{d})_{R}^{T}
$$


where the graded equal-time commutation relations for two fields is

$$
Q_{i}^{\alpha}(\mathbf{x}) Q_{k}^{\beta \dagger}(\mathbf{y})-(-)^{\eta_{i} \eta_{k}} Q_{k}^{\beta \dagger}(\mathbf{y}) Q_{i}^{\alpha}(\mathbf{x})=\delta^{\alpha \beta} \delta_{i k} \delta^{3}(\mathbf{x}-\mathbf{y})
$$

where $\alpha, \beta$ are spin-indices and $i, k$ are flavor indices. The objects $\eta_{k}$ correspond to the parity of the component of $Q_{k}$, with $\eta_{k}=+1$ for $k=1,2,3,4$ and $\eta_{k}=0$ for $k=5,6$, and the graded commutation relations for two $Q$ 's or two $Q^{\dagger}$ 's are analogous. The $Q_{L, R}$ in eq. (2) transform in the fundamental representation of $S U(4 \mid 2)_{L, R}$ respectively. The ground floor of $Q_{L}$ transforms as a $(\mathbf{4}, \mathbf{1})$ of $S U(4)_{q L} \otimes S U(2)_{\tilde{q} L}$ while the first floor transforms as $(\mathbf{1}, \mathbf{2})$, and the right handed field $Q_{R}$ transforms analogously.

In the absence of quark masses, $m_{Q}=0$, the Lagrange density in eq. (1) has a graded symmetry $U(4 \mid 2)_{L} \otimes U(4 \mid 2)_{R}$, where the left- and right-handed quark fields transform as $Q_{L} \rightarrow U_{L} Q_{L}$ and $Q_{R} \rightarrow U_{R} Q_{R}$ respectively. The strong anomaly reduces the symmetry of the theory, which can be taken to be $S U(4 \mid 2)_{L} \otimes S U(4 \mid 2)_{R} \otimes U(1)_{V}$ [7]. It is assumed that this symmetry is spontaneously broken according to the pattern $S U(4 \mid 2)_{L} \otimes S U(4 \mid 2)_{R} \otimes U(1)_{V} \rightarrow$ $S U(4 \mid 2)_{V} \otimes U(1)_{V}$ so that an identification with QCD can be made.

The mass-matrix, $m_{Q}$, has entries $m_{Q}=\operatorname{diag}\left(m_{u}, m_{d}, m_{j}, m_{l}, m_{u}, m_{d}\right)$, i.e. $m_{\tilde{u}}=m_{u}$ and $m_{\tilde{d}}=m_{d}$, so that the contribution to the determinant in the path integral from integrating over the $q_{V}$ and the $\tilde{q}$ exactly cancel, leaving the contribution from the $q_{\text {sea }}$ alone.

\section{A. The Pseudo-Goldstone Bosons}

The strong interaction dynamics of the pseudo-Goldstone bosons are described at leading order (LO) in PQ $\chi \mathrm{PT}$ by a Lagrange density of the form [6],

$$
\mathcal{L}=\frac{f^{2}}{8} \operatorname{str}\left[\partial^{\mu} \Sigma^{\dagger} \partial_{\mu} \Sigma\right]+\lambda \frac{f^{2}}{4} \operatorname{str}\left[m_{Q} \Sigma^{\dagger}+m_{Q} \Sigma\right]+\alpha_{\Phi} \partial^{\mu} \Phi_{0} \partial_{\mu} \Phi_{0}-m_{0}^{2} \Phi_{0}^{2}
$$

where $\alpha_{\Phi}$ and $m_{0}$ are quantities that do not vanish in the chiral limit. The meson field is incorporated in $\Sigma$ via

$$
\Sigma=\exp \left(\frac{2 i \Phi}{f}\right)=\xi^{2} \quad, \quad \Phi=\left(\begin{array}{cc}
M & \chi_{\tilde{M}}^{\dagger} \\
\chi & \tilde{M}
\end{array}\right)
$$

where $M$ and $\tilde{M}$ are matrices containing bosonic mesons while $\chi$ and $\chi^{\dagger}$ are matrices containing fermionic mesons, with

$M=\left(\begin{array}{cccc}\eta_{u} & \pi^{+} & J^{0} & L^{+} \\ \pi^{-} & \eta_{d} & J^{-} & L^{0} \\ \bar{J}^{0} & J^{+} & \eta_{j} & Y_{j l}^{+} \\ L^{-} & \bar{L}^{0} & Y_{j l}^{-} & \eta_{l}\end{array}\right), \tilde{M}=\left(\begin{array}{cc}\tilde{\eta}_{u} & \tilde{\pi}^{+} \\ \tilde{\pi}^{-} & \tilde{\eta}_{d}\end{array}\right), \chi=\left(\begin{array}{cccc}\chi_{\eta_{u}} & \chi_{\pi^{+}} & \chi_{J^{0}} & \chi_{L^{+}} \\ \chi_{\pi^{-}} & \chi_{\eta_{d}} & \chi_{J^{-}} & \chi_{L^{0}}\end{array}\right)$

where the upper $2 \times 2$ block of $M$ is the usual triplet plus singlet of pseudo-scalar mesons while the remaining entries correspond to mesons formed with the sea-quarks. The convention we use corresponds to $f \sim 132 \mathrm{MeV}$, and the charge assignments have been made using an electromagnetic charge matrix, $\mathcal{Q}^{(P Q)}=\frac{1}{3} \operatorname{diag}(2,-1,2,-1,2,-1)$. 
The singlet field is defined to be $\Phi_{0}=\operatorname{str}(\Phi) / \sqrt{2}$, and its mass $m_{0}$ can be taken to be of order the scale of chiral symmetry breaking, $m_{0} \rightarrow \Lambda_{\chi}$ [7]. In taking this limit, one finds that the $\eta$ two-point functions deviate from simple single-poles. The $\eta_{a} \eta_{b}$ propagator is found to be

$$
\mathcal{G}_{\eta_{a} \eta_{b}}=\frac{i \delta^{a b}}{q^{2}-m_{\eta_{a}}^{2}+i \epsilon}-\frac{i}{2} \frac{\left(q^{2}-m_{j j}^{2}\right)\left(q^{2}-m_{l l}^{2}\right)}{\left(q^{2}-m_{\eta_{a}}^{2}+i \epsilon\right)\left(q^{2}-m_{\eta_{b}}^{2}+i \epsilon\right)\left(q^{2}-\frac{1}{2}\left(m_{j j}^{2}+m_{l l}^{2}\right)+i \epsilon\right)},
$$

where $m_{a b}$ is the mass of the meson composed of (anti)-quarks of flavor $a$ and $b$. This can be compactly written as

$$
\mathcal{G}_{\eta_{a} \eta_{b}}=\delta^{a b} P_{a}+\mathcal{H}_{a b}\left(P_{a}, P_{b}, P_{X}\right)
$$

where

$$
\begin{aligned}
& P_{a}=\frac{i}{q^{2}-m_{\eta_{a}}^{2}+i \epsilon} \quad, \quad P_{b}=\frac{i}{q^{2}-m_{\eta_{b}}^{2}+i \epsilon} \quad, \quad P_{X}=\frac{i}{q^{2}-m_{X}^{2}+i \epsilon} \\
& \mathcal{H}_{a b}(A, B, C)=-\frac{1}{2}\left[\frac{\left(m_{j j}^{2}-m_{\eta_{a}}^{2}\right)\left(m_{l l}^{2}-m_{\eta_{a}}^{2}\right)}{\left(m_{\eta_{a}}^{2}-m_{\eta_{b}}^{2}\right)\left(m_{\eta_{a}}^{2}-m_{X}^{2}\right)} A-\frac{\left(m_{j j}^{2}-m_{\eta_{b}}^{2}\right)\left(m_{l l}^{2}-m_{\eta_{b}}^{2}\right)}{\left(m_{\eta_{a}}^{2}-m_{\eta_{b}}^{2}\right)\left(m_{\eta_{b}}^{2}-m_{X}^{2}\right)} B\right. \\
& \left.\quad+\frac{\left(m_{X}^{2}-m_{j j}^{2}\right)\left(m_{X}^{2}-m_{l l}^{2}\right)}{\left(m_{X}^{2}-m_{\eta_{a}}^{2}\right)\left(m_{X}^{2}-m_{\eta_{b}}^{2}\right)} C\right],
\end{aligned}
$$

where the mass, $m_{X}$, is given by $m_{X}^{2}=\frac{1}{2}\left(m_{j j}^{2}+m_{l l}^{2}\right)$. One important implication of this form for the singlet propagator is that the nucleon-nucleon potential falls off exponentially at long distances, as opposed to Yukawa-like behavior, away from the QCD-limit [9].

The tree-level masses resulting from the Lagrange density in eq. (4) are

$$
\begin{aligned}
& m_{\pi^{ \pm}}^{2}=m_{u d}^{2}=\lambda\left(m_{u}+m_{d}\right) \quad, \quad m_{\eta_{u}}^{2}=m_{u u}^{2}=2 \lambda m_{u} \\
& m_{J^{0}}^{2}=m_{j u}^{2}=\lambda\left(m_{j}+m_{u}\right)
\end{aligned}
$$

and so forth.

\section{B. The Nucleons and $\Delta$-Resonances}

It is straightforward to include the proton, neutron, and $\Delta$-resonances into PQ $\chi \mathrm{PT}$ 幽《]. An interpolating field that has non-zero overlap with the nucleons (when the $i j k$ indices are restricted to 1,2$)$ is $[4]$

$$
\mathcal{B}_{i j k}^{\gamma} \sim\left[Q_{i}^{\alpha, a} Q_{j}^{\beta, b} Q_{k}^{\gamma, c}-Q_{i}^{\alpha, a} Q_{j}^{\gamma, c} Q_{k}^{\beta, b}\right] \epsilon_{a b c}\left(C \gamma_{5}\right)_{\alpha \beta} \quad,
$$

where $C$ is the charge conjugation operator, $a, b, c$ are color indices and $\alpha, \beta, \gamma$ are Dirac indices. Dropping the Dirac index, one finds that under the interchange of flavor indices 团,

$$
\mathcal{B}_{i j k}=(-)^{1+\eta_{j} \eta_{k}} \mathcal{B}_{i k j}, \mathcal{B}_{i j k}+(-)^{1+\eta_{i} \eta_{j}} \mathcal{B}_{j i k}+(-)^{1+\eta_{i} \eta_{j}+\eta_{j} \eta_{k}+\eta_{k} \eta_{i}} \mathcal{B}_{k j i}=0
$$

In analogy with $\mathrm{QCD}$, we consider the transformation of $\mathcal{B}_{i j k}$ under $S U(4 \mid 2)_{V}$ transformations, and using the graded relation 


$$
Q_{i} U^{j}{ }_{k}=(-)^{\eta_{i}\left(\eta_{j}+\eta_{k}\right)} U^{j}{ }_{k} Q_{i},
$$

in eq. (11), it is straightforward to show that [1]

$$
\mathcal{B}_{i j k} \rightarrow(-)^{\eta_{l}\left(\eta_{j}+\eta_{m}\right)+\left(\eta_{l}+\eta_{m}\right)\left(\eta_{k}+\eta_{n}\right)} U_{i}^{l} U_{j}^{m} U_{k}^{n} \mathcal{B}_{l m n},
$$

where $\mathcal{B}_{i j k}$ describes a $\mathbf{7 0}$ dimensional representation of $S U(4 \mid 2)_{V}$.

It is convenient to decompose the irreducible representations of $S U(4 \mid 2)_{V}$ into irreducible representations of $S U(2)_{\mathrm{val}} \otimes S U(2)_{\mathrm{sea}} \otimes S U(2)_{\tilde{q}}$ [8, 10, 12]. The subscript denotes where the $S U(2)$ acts, either on the $q_{V}$, the $q_{\text {sea }}$, or the $\tilde{q}$. In order to locate a particular baryon in the irreducible representation we employ the terminology of Ref. [8]]: ground floor, first floor, second floor and so on, as it is common in the description of super-algebra multiplets. The ground floor contains all the baryons that do not contain a bosonic quark, the first floor contains all baryons that contain one bosonic quark, the second floor contains all baryons that contain two bosonic quarks, and the third floor contains the baryons that are comprised entirely of bosonic quarks. As a way of distinguishing between baryons containing some number of valence and sea quarks, we use "levels" [B]. Level A consists of baryons containing no sea quarks, level B consists of baryons containing one sea quarks, level C consists of baryons containing two sea quarks, and level D consists of baryons composed only of sea quarks.

The ground floor of level A of the 70-dimensional representation contains nucleons, comprised of three valence quarks, $q_{V} q_{V} q_{V}$, and is therefore a $(\mathbf{2}, \mathbf{1}, \mathbf{1})$ of $S U(2)_{\text {val }} \otimes S U(2)_{\text {sea }} \otimes$ $S U(2)_{\tilde{q}}$. The nucleons are embedded as

$$
\mathcal{B}_{a b c}=\frac{1}{\sqrt{6}}\left(\epsilon_{a b} N_{c}+\epsilon_{a c} N_{b}\right),
$$

where the indices are restricted to take the values $a, b, c=1,2$ only. The nucleon doublet is

$$
N=\left(\begin{array}{l}
p \\
n
\end{array}\right) \text {. }
$$

The first floor of level A of the 70-dimensional representation contains baryons that are composed of two valence quarks and one ghost-quark, $\tilde{q} q_{V} q_{V}$, and therefore transforms as $(\mathbf{3}, \mathbf{1}, \mathbf{2}) \oplus(\mathbf{1}, \mathbf{1}, \mathbf{2})$ of $S U(2)_{\text {val }} \otimes S U(2)_{\text {sea }} \otimes S U(2)_{\tilde{q}}$. The tensor representation $\tilde{a}_{\tilde{s}} \tilde{s}_{a b}$ of the $(\mathbf{3}, \mathbf{1}, \mathbf{2})$ multiplet, and $\tilde{a} \tilde{t}$ of the $(\mathbf{1}, \mathbf{1}, \mathbf{2})$ multiplet, where $\tilde{a}=1,2$ runs over the $\tilde{q}$ indices and $a, b=1,2$ run over the $q_{V}$ indices, have baryon assignments

$$
\tilde{a} \tilde{s}_{11}=\tilde{\Sigma}_{\tilde{a}}^{+1}, \quad \tilde{a}_{12}=\tilde{a}_{a} \tilde{s}_{21}=\frac{1}{\sqrt{2}} \tilde{\Sigma}_{\tilde{a}}^{0}, \quad \tilde{a}_{\tilde{s}_{22}}=\tilde{\Sigma}_{\tilde{a}}^{-1}, \quad \tilde{a} \tilde{t}=\tilde{\Lambda}_{\tilde{a}}^{0} .
$$

The right superscript denotes the third component of $q_{V}$-isospin, while the left subscript denotes the $\tilde{q}$ flavor. The ground floor of level $\mathrm{B}$ of the 70-dimensional representation contains baryons that are composed of two valence quarks and one sea quark, $q_{V} q_{V} q_{\text {sea }}$, and therefore transforms as $(\mathbf{3}, \mathbf{2}, \mathbf{1}) \oplus(\mathbf{1}, \mathbf{2}, \mathbf{1})$ of $S U(2)_{\text {val }} \otimes S U(2)_{\text {sea }} \otimes S U(2)_{\tilde{q}}$. The tensor representation ${ }_{a} s_{b c}$ of the $(\mathbf{3}, \mathbf{2}, \mathbf{1})$ multiplet, and ${ }_{a} t$ of the $(\mathbf{1}, \mathbf{2}, \mathbf{1})$ multiplet, where $a=1,2$ runs over the $q_{\text {sea }}$ indices and $b, c=1,2$ run over the $q_{V}$ indices, have baryon assignment

$$
{ }_{a} s_{11}=\Sigma_{a}^{+1},{ }_{a} s_{12}={ }_{a} s_{21}=\frac{1}{\sqrt{2}} \Sigma_{a}^{0},{ }_{a} s_{22}=\Sigma_{a}^{-1},{ }_{a} t=\Lambda_{a}^{0} .
$$


The $\tilde{a}_{a b}, \tilde{a} \tilde{t},{ }_{a} s_{a b}$, and ${ }_{a} t$ are uniquely embedded into $\mathcal{B}_{i j k}$ (up to field redefinitions), constrained by the relations in eq. (12):

$$
\begin{aligned}
& \mathcal{B}_{i j k}=-\sqrt{\frac{2}{3}}{ }_{i-2} s_{j k} \quad \text { for } i=3,4 \text { and } j, k=1,2 \\
& \mathcal{B}_{i j k}=\frac{1}{2}{ }_{j-2} t \varepsilon_{i k}+\frac{1}{\sqrt{6}}{ }_{j-2} s_{i k} \quad \text { for } j=3,4 \text { and } i, k=1,2 \\
& \mathcal{B}_{i j k}=\frac{1}{2}{ }_{k-2} t \varepsilon_{i j}+\frac{1}{\sqrt{6}}{ }_{k-2} s_{i j} \quad \text { for } \quad k=3,4 \text { and } i, j=1,2 \\
& \mathcal{B}_{i j k}=\sqrt{\frac{2}{3}}{ }_{i-4} \tilde{s}_{j k} \quad \text { for } i=5,6 \text { and } j, k=1,2 \\
& \mathcal{B}_{i j k}=\frac{1}{2}{ }_{j-4} \tilde{t} \varepsilon_{i k}+\frac{1}{\sqrt{6}}{ }_{j-4} \tilde{s}_{i k} \quad \text { for } j=5,6 \text { and } i, k=1,2 \\
& \mathcal{B}_{i j k}=-\frac{1}{2}{ }_{k-4} \tilde{t} \varepsilon_{i j}-\frac{1}{\sqrt{6}}{ }_{k-4} \tilde{s}_{i j} \quad \text { for } k=5,6 \text { and } i, j=1,2 .
\end{aligned}
$$

We do not construct the remaining floors and levels of the $\mathbf{7 0}$ as we are only interested in one-loop contributions to observables with nucleons in the asymptotic states.

As the mass splitting between the $\Delta$-resonances and nucleons in QCD is much less than the scale of chiral symmetry breaking $\left(\Lambda_{\chi} \sim 1 \mathrm{GeV}\right)$ the $\Delta$-resonances must be included as a dynamical field in order to have a theory where the natural scale of higher order interactions is set by $\Lambda_{\chi}$. An interpolating field that contains the spin- $\frac{3}{2} \Delta$-resonances as the ground floor is [4]

$$
\mathcal{T}_{i j k}^{\alpha, \mu} \sim\left[Q_{i}^{\alpha, a} Q_{j}^{\beta, b} Q_{k}^{\gamma, c}+Q_{i}^{\beta, b} Q_{j}^{\gamma, c} Q_{k}^{\alpha, a}+Q_{i}^{\gamma, c} Q_{j}^{\alpha, a} Q_{k}^{\beta, b}\right] \varepsilon_{a b c}\left(C \gamma^{\mu}\right)_{\beta \gamma} \quad,
$$

where the indices $i, j, k$ run from 1 to 6 . Neglecting Dirac indices, one finds that under the interchange of flavor indices [4]

$$
\mathcal{T}_{i j k}=(-)^{1+\eta_{i} \eta_{j}} \mathcal{T}_{j i k}=(-)^{1+\eta_{j} \eta_{k}} \mathcal{T}_{i k j}
$$

$\mathcal{T}_{i j k}$ describes a 44 dimensional representation of $S U(4 \mid 2)_{V}$, which has the ground floor of level A transforming as $(\mathbf{4}, \mathbf{1}, \mathbf{1})$ under $S U(2)_{\text {val }} \otimes S U(2)_{\text {sea }} \otimes S U(2)_{\tilde{q}}$ with $\mathcal{T}_{a b c}=T_{a b c}$, when the indices are restricted to take the values $a, b, c=1,2$, and where $T_{a b c}$ is the totally symmetric tensor containing the $\Delta$-resonances,

$$
T_{111}=\Delta^{++}, \quad T_{112}=\frac{1}{\sqrt{3}} \Delta^{+}, T_{122}=\frac{1}{\sqrt{3}} \Delta^{0}, T_{222}=\Delta^{-} .
$$

The first floor of level A of the $\mathbf{4 4}$ transforms as a $(\mathbf{3}, \mathbf{1}, \mathbf{2})$ under $S U(2)_{\mathrm{val}} \otimes S U(2)_{\mathrm{sea}} \otimes S U(2)_{\tilde{q}}$ which has a tensor representation, $\tilde{a} \tilde{x}_{i j}$, with baryon assignment

$$
\tilde{a} \tilde{x}_{11}=\tilde{\Sigma}_{\tilde{a}}^{*,+1} \quad, \quad \tilde{a}_{12}={ }_{\tilde{a}} \tilde{x}_{21}=\frac{1}{\sqrt{2}} \tilde{\Sigma}_{\tilde{a}}^{*, 0} \quad, \quad \tilde{a}_{22}=\tilde{\Sigma}_{\tilde{a}}^{*,-1}
$$

Similarly, the ground floor of level B of the 44 transforms as a $(\mathbf{3}, \mathbf{2}, \mathbf{1})$ under $S U(2)_{\text {val }} \otimes$ $S U(2)_{\text {sea }} \otimes S U(2)_{\tilde{q}}$ which has a tensor representation, ${ }_{a} x_{i j}$, with baryon assignment 


$$
{ }_{a} x_{11}=\Sigma_{a}^{*,+1},{ }_{a} x_{12}={ }_{a} x_{21}=\frac{1}{\sqrt{2}} \Sigma_{a}^{*, 0},{ }_{a} x_{22}=\Sigma_{a}^{*,-1} .
$$

The embedding of ${ }_{a} x_{i j}$ into $\mathcal{T}_{i j k}$ is unique (up to field redefinitions), constrained by the symmetry properties in eq. (21):

$$
\begin{array}{lll}
\mathcal{T}_{i j k}=+\frac{1}{\sqrt{3}}{ }_{i-2} x_{j k} & \text { for } & i=3,4 \text { and } j, k=1,2 \\
\mathcal{T}_{i j k}=+\frac{1}{\sqrt{3}}{ }_{j-2} x_{i k} & \text { for } & j=3,4 \text { and } i, k=1,2 \\
\mathcal{T}_{i j k}=+\frac{1}{\sqrt{3}}{ }_{k-2} x_{i j} & \text { for } & k=3,4 \text { and } i, j=1,2 \\
\mathcal{T}_{i j k}=+\frac{1}{\sqrt{3}}{ }_{i-4} \tilde{x}_{j k} & \text { for } & i=5,6 \text { and } j, k=1,2 \\
\mathcal{T}_{i j k}=-\frac{1}{\sqrt{3}}{ }_{j-4} \tilde{x}_{i k} & \text { for } & j=5,6 \text { and } i, k=1,2 \\
\mathcal{T}_{i j k}=+\frac{1}{\sqrt{3}}{ }_{k-4} \tilde{x}_{i j} & \text { for } & k=5,6
\end{array}
$$

Again, we do not explicitly construct the second and third floor baryons of the $\mathbf{4 4}$.

\section{Lagrange Density for the Nucleons and $\Delta$-Resonances}

The free Lagrange density for the $\mathcal{B}_{i j k}$ and $\mathcal{T}_{i j k}$ fields is [4], at LO in the heavy baryon expansion [13 17],

$$
\begin{aligned}
\mathcal{L} & =i(\overline{\mathcal{B}} v \cdot \mathcal{D} \mathcal{B})+2 \alpha_{M}^{(\mathrm{PQ})}\left(\overline{\mathcal{B}} \mathcal{B} \mathcal{M}_{+}\right)+2 \beta_{M}^{(\mathrm{PQ})}\left(\overline{\mathcal{B}} \mathcal{M}_{+} \mathcal{B}\right)+2 \sigma_{M}^{(\mathrm{PQ})}(\overline{\mathcal{B}} \mathcal{B}) \operatorname{str}\left(\mathcal{M}_{+}\right) \\
& -i\left(\overline{\mathcal{T}}^{\mu} v \cdot \mathcal{D} \mathcal{T}_{\mu}\right)+\Delta\left(\overline{\mathcal{T}}^{\mu} \mathcal{T}_{\mu}\right)-2 \gamma_{M}^{(\mathrm{PQ})}\left(\overline{\mathcal{T}}^{\mu} \mathcal{M}_{+} \mathcal{T}_{\mu}\right)-2 \bar{\sigma}_{M}^{(\mathrm{PQ})}\left(\overline{\mathcal{T}}^{\mu} \mathcal{T}_{\mu}\right) \operatorname{str}\left(\mathcal{M}_{+}\right)
\end{aligned}
$$

where $\Delta$ is the mass splitting between the $\mathbf{7 0}$ and the $\mathbf{4 4}, \mathcal{M}_{+}=\frac{1}{2}\left(\xi^{\dagger} m_{Q} \xi^{\dagger}+\xi m_{Q} \xi\right)$, and $\xi=\sqrt{\Sigma}$. The brackets, ( ) denote contraction of Lorentz and flavor indices as defined in Ref. [⿴囗⿴囗十 . For a matrix $\Gamma_{\beta}^{\alpha}$ acting in spin-space, and a matrix $Y_{i j}$ that acts in flavor-space, the required contractions are [4]

$$
\begin{aligned}
(\overline{\mathcal{B}} \Gamma \mathcal{B}) & =\overline{\mathcal{B}}^{\alpha, k j i} \Gamma_{\alpha}^{\beta} \mathcal{B}_{i j k, \beta}, \quad\left(\overline{\mathcal{T}}^{\mu} \Gamma \mathcal{T}_{\mu}\right)=\overline{\mathcal{T}}^{\mu \alpha, k j i} \Gamma_{\alpha}^{\beta} \mathcal{T}_{i j k, \beta \mu} \\
(\overline{\mathcal{B}} \Gamma Y \mathcal{B}) & =\overline{\mathcal{B}}^{\alpha, k j i} \Gamma_{\alpha}^{\beta} Y_{i}^{l} \mathcal{B}_{l j k, \beta}, \quad\left(\overline{\mathcal{T}}^{\mu} \Gamma Y \mathcal{T}_{\mu}\right)=\overline{\mathcal{T}}^{\mu \alpha, k j i} \Gamma_{\alpha}^{\beta} Y_{i}{ }^{l} \mathcal{T}_{l j k, \beta \mu} \\
(\overline{\mathcal{B}} \Gamma \mathcal{B} Y) & =(-)^{\left(\eta_{i}+\eta_{j}\right)\left(\eta_{k}+\eta_{n}\right)} \overline{\mathcal{B}}^{\alpha, k j i} \Gamma_{\alpha}^{\beta} Y_{k}{ }^{n} \mathcal{B}_{i j n, \beta} \\
\left(\overline{\mathcal{B}} \Gamma Y^{\mu} \mathcal{T}_{\mu}\right) & =\overline{\mathcal{B}}^{\alpha, k j i} \Gamma_{\alpha}^{\beta}\left(Y^{\mu}\right)_{i}^{l} \mathcal{T}_{l j k, \beta \mu},
\end{aligned}
$$

where $\overline{\mathcal{B}}$ and $\overline{\mathcal{T}}$ transform the same way, e.g.

$$
\overline{\mathcal{B}}^{k j i} \rightarrow(-)^{\eta_{l}\left(\eta_{j}+\eta_{m}\right)+\left(\eta_{l}+\eta_{m}\right)\left(\eta_{k}+\eta_{n}\right)} \overline{\mathcal{B}}^{n m l} U_{n}{ }^{k \dagger} U_{m}^{j \dagger} U_{l}^{i \dagger}
$$

The covariant derivative acting on either the $\mathcal{B}$ or $\mathcal{T}$ fields has the form 


$$
\left(\mathcal{D}^{\mu} \mathcal{B}\right)_{i j k}=\partial^{\mu} \mathcal{B}_{i j k}+\left(V^{\mu}\right)_{i}^{l} \mathcal{B}_{l j k}+(-)^{\eta_{i}\left(\eta_{j}+\eta_{m}\right)}\left(V^{\mu}\right)_{j}^{m} \mathcal{B}_{i m k}+(-)^{\left(\eta_{i}+\eta_{j}\right)\left(\eta_{k}+\eta_{n}\right)}\left(V^{\mu}\right)_{k}^{n} \mathcal{B}_{i j n}
$$

where the vector and axial-vector meson fields are

$$
V^{\mu}=\frac{1}{2}\left(\xi \partial^{\mu} \xi^{\dagger}+\xi^{\dagger} \partial^{\mu} \xi\right), A^{\mu}=\frac{i}{2}\left(\xi \partial^{\mu} \xi^{\dagger}-\xi^{\dagger} \partial^{\mu} \xi\right) .
$$

By restricting ourselves to the $q_{V} q_{V} q_{V}$ sector, we can make a comparison between the LO partially-quenched free Lagrange density and that of QCD, which has fewer free parameters,

$$
\begin{aligned}
\mathcal{L}^{Q C D} & =i \bar{N} v \cdot \mathcal{D} N+2 \alpha_{M} \bar{N} \mathcal{M}_{+}^{\mathrm{QCD}} N+2 \sigma_{M} \bar{N} N \operatorname{tr}\left[\mathcal{M}_{+}^{\mathrm{QCD}}\right] \\
& -i \bar{T}^{\mu} v \cdot \mathcal{D} T_{\mu}+\Delta \bar{T}^{\mu} T_{\mu}-2 \gamma_{M} \bar{T}^{\mu} \mathcal{M}_{+}^{\mathrm{QCD}} T_{\mu}-2 \bar{\sigma}_{M} \bar{T}^{\mu} T_{\mu} \operatorname{tr}\left[\mathcal{M}_{+}^{\mathrm{QCD}}\right],
\end{aligned}
$$

where the QCD chirally-invariant mass operator is $\mathcal{M}_{+}^{\mathrm{QCD}}=\frac{1}{2}\left(\xi^{\dagger} m_{q} \xi^{\dagger}+\xi m_{q} \xi\right)$, where $m_{q}=\operatorname{diag}\left(m_{u}, m_{d}\right)$, and $\xi$ is the $\mathrm{QCD}$ version of the matrix defined in eq. (5). The QCD parameters and the PQQCD parameters are related by,

$$
\begin{aligned}
& \alpha_{M}=\frac{2}{3} \alpha_{M}^{(P Q)}-\frac{1}{3} \beta_{M}^{(P Q)}, \sigma_{M}=\sigma_{M}^{(P Q)}+\frac{1}{6} \alpha_{M}^{(P Q)}+\frac{2}{3} \beta_{M}^{(P Q)} \\
& \gamma_{M}=\gamma_{M}^{(P Q)}, \quad \bar{\sigma}_{M}=\bar{\sigma}_{M}^{(P Q)} .
\end{aligned}
$$

The Lagrange density describing the interactions of the $\mathbf{7 0}$ and $\mathbf{4 4}$ with the pseudoGoldstone bosons at $\mathrm{LO}$ in the chiral expansion is [4],

$$
\begin{aligned}
\mathcal{L}= & 2 \alpha\left(\overline{\mathcal{B}} S^{\mu} \mathcal{B} A_{\mu}\right)+2 \beta\left(\overline{\mathcal{B}} S^{\mu} A_{\mu} \mathcal{B}\right)+2 \mathcal{H}\left(\overline{\mathcal{T}}^{\nu} S^{\mu} A_{\mu} \mathcal{T}_{\nu}\right) \\
& +\sqrt{\frac{3}{2}} \mathcal{C}\left[\left(\overline{\mathcal{T}}^{\nu} A_{\nu} \mathcal{B}\right)+\left(\overline{\mathcal{B}} A_{\nu} \mathcal{T}^{\nu}\right)\right]
\end{aligned}
$$

where $S^{\mu}$ is the covariant spin-vector [13 15]. Restricting ourselves to the $q_{V} q_{V} q_{V}$ sector, we can compare eq. (33) with the LO interaction Lagrange density of QCD,

$$
\begin{aligned}
\mathcal{L}= & 2 g_{A} \bar{N} S^{\mu} A_{\mu} N+g_{1} \bar{N} S^{\mu} N \operatorname{tr}\left[A_{\mu}\right]+g_{\Delta N}\left[\bar{T}^{a b c, \nu} A_{a, \nu}^{d} N_{b} \epsilon_{c d}+\text { h.c. }\right] \\
& +2 g_{\Delta \Delta} \bar{T}^{\nu} S^{\mu} A_{\mu} T_{\nu}+2 g_{X} \bar{T}^{\nu} S^{\mu} T_{\nu} \operatorname{tr}\left[A_{\mu}\right]
\end{aligned}
$$

and find that at tree-level

$$
\alpha=\frac{4}{3} g_{A}+\frac{1}{3} g_{1} \quad, \quad \beta=\frac{2}{3} g_{1}-\frac{1}{3} g_{A} \quad, \quad \mathcal{H}=g_{\Delta \Delta} \quad, \quad \mathcal{C}=-g_{\Delta N},
$$

with $g_{X}=0$. Considering only the nucleons, and decomposing the Lagrange density in eq. (34) into the mass eigenstates of the isospin-symmetric limit, $\pi^{ \pm}, \pi^{0}$ and $\eta$, we have

$$
\mathcal{L}=2 g_{A} \bar{N} S^{\mu} \tilde{A}_{\mu} N+\frac{\sqrt{2}}{f}\left(g_{A}+g_{1}\right) \bar{N} S^{\mu} N \partial_{\mu} \eta,
$$

where $\tilde{A}_{\mu}$ is the axial-vector field of pions only (excluding the isosinglet meson). In the isospin symmetric limit, with the mass of the $\eta$ being of order $\sim \Lambda_{\chi}$, all expressions must be independent of the coupling $g_{1}$. 


\section{NUCLEON MASSES}

The mass of the $i$-th nucleon has a chiral expansion

$$
M_{i}=M_{0}(\mu)-M_{i}^{(1)}(\mu)-M_{i}^{(3 / 2)}(\mu)+\ldots,
$$

where a term $M_{i}^{(\alpha)}$ denotes a contribution of order $m_{Q}^{\alpha}$, and $i=p, n$. The nucleon mass is dominated by a term in the PQ $\chi \mathrm{PT}$ Lagrange density, $M_{0}$, that is independent of $m_{Q}$. Each of the contributions in eq. (37) depends upon the scale chosen to renormalize the theory. While at LO and next-to-leading order (NLO) in the chiral expansion, the objects $M_{0}$ and $M_{i}^{(1)}$ are scale independent, at one-loop level they are required to be scale dependent. The leading dependence upon $m_{Q}$, occurring at order $\mathcal{O}\left(m_{Q}\right)$, is due to the terms in eq. (26) with coefficients $\alpha_{M}^{(P Q)}, \beta_{M}^{(P Q)}$ and $\sigma_{M}^{(P Q)}$, each of which must be determined from lattice simulations. The leading non-analytic dependence upon $m_{Q}$ arises from the one-loop diagrams shown in Fig. 1, and we find the contributions to the proton mass are

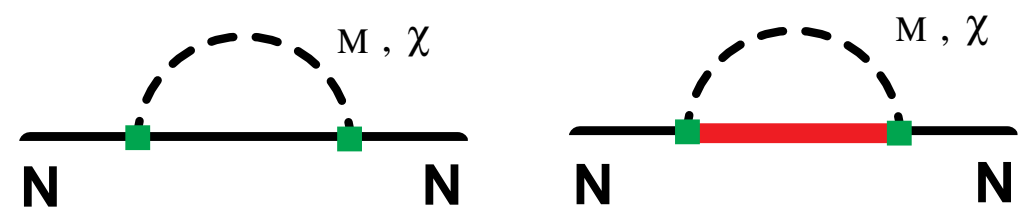

FIG. 1. One-loop graphs that give contributions of the form $\sim m_{Q}^{3 / 2}$ to the masses of the proton and neutron. A solid, thick-solid and dashed line denote a 70-nucleon, 44-resonance, and a meson, respectively. The solid-squares denote an axial coupling.

$$
\begin{aligned}
M_{p}^{(1)} & =\frac{1}{3} m_{u}\left(5 \alpha_{M}^{(P Q)}+2 \beta_{M}^{(P Q)}\right)+\frac{1}{3} m_{d}\left(\alpha_{M}^{(P Q)}+4 \beta_{M}^{(P Q)}\right)+2 \sigma_{M}^{(P Q)}\left(m_{j}+m_{l}\right) \\
M_{p}^{(3 / 2)} & =\frac{1}{8 \pi f^{2}}\left(\frac{g_{A}^{2}}{3}\left[m_{u u}^{3}+m_{u d}^{3}+2 m_{j u}^{3}+2 m_{l u}^{3}+3 G_{\eta_{u}, \eta_{u}}\right]\right. \\
& +\frac{g_{1}^{2}}{12}\left[m_{u u}^{3}-5 m_{u d}^{3}+3 m_{j d}^{3}+2 m_{j u}^{3}+3 m_{l d}^{3}+2 m_{l u}^{3}+3 G_{\eta_{u}, \eta_{u}}+6 G_{\eta_{u}, \eta_{d}}+3 G_{\eta_{d}, \eta_{d}}\right] \\
& +\frac{g_{A} g_{1}}{3}\left[m_{j u}^{3}+m_{l u}^{3}-m_{u d}^{3}+2 m_{u u}^{3}+3 G_{\eta_{u}, \eta_{d}}+3 G_{\eta_{u}, \eta_{u}}\right] \\
+ & \left.\frac{g_{\Delta N}^{2}}{9 \pi}\left[5 F_{u d}+F_{u u}+F_{j u}+F_{l u}+2 F_{j d}+2 F_{l d}+2 E_{\eta_{d}, \eta_{d}}+2 E_{\eta_{u}, \eta_{u}}-4 E_{\eta_{u}, \eta_{d}}\right]\right)
\end{aligned}
$$

where the function $F_{c}=F\left(m_{c}, \Delta, \mu\right)$ is

$$
\begin{aligned}
F(m, \Delta, \mu) & =\left(m^{2}-\Delta^{2}\right)\left(\sqrt{\Delta^{2}-m^{2}} \log \left(\frac{\Delta-\sqrt{\Delta^{2}-m^{2}+i \epsilon}}{\Delta+\sqrt{\Delta^{2}-m^{2}+i \epsilon}}\right)-\Delta \log \left(\frac{m^{2}}{\mu^{2}}\right)\right) \\
& -\frac{1}{2} \Delta m^{2} \log \left(\frac{m^{2}}{\mu^{2}}\right) .
\end{aligned}
$$


The functions $G_{\eta_{a}, \eta_{b}}$ and $E_{\eta_{a}, \eta_{b}}$ are $G_{\eta_{a}, \eta_{b}}=\mathcal{H}_{\eta_{a} \eta_{b}}\left(m_{\eta_{a}}^{3}, m_{\eta_{b}}^{3}, m_{X}^{3}\right)$ and $E_{\eta_{a}, \eta_{b}}=$ $\mathcal{H}_{\eta_{a} \eta_{b}}\left(F_{\eta_{a}}, F_{\eta_{b}}, F_{X}\right)$, respectively, where the function $\mathcal{H}_{\eta_{a} \eta_{b}}$ is given in eq. (9). The contributions to the neutron mass are

$$
\begin{aligned}
M_{n}^{(1)} & =\frac{1}{3} m_{u}\left(\alpha_{M}^{(P Q)}+4 \beta_{M}^{(P Q)}\right)+\frac{1}{3} m_{d}\left(5 \alpha_{M}^{(P Q)}+2 \beta_{M}^{(P Q)}\right)+2 \sigma_{M}^{(P Q)}\left(m_{j}+m_{l}\right) \\
M_{n}^{(3 / 2)} & =\frac{1}{8 \pi f^{2}}\left(\frac{g_{A}^{2}}{3}\left[m_{d d}^{3}+m_{u d}^{3}+2 m_{j d}^{3}+2 m_{l d}^{3}+3 G_{\eta_{d}, \eta_{d}}\right]\right. \\
& +\frac{g_{1}^{2}}{12}\left[m_{d d}^{3}-5 m_{u d}^{3}+2 m_{j d}^{3}+3 m_{j u}^{3}+2 m_{l d}^{3}+3 m_{l u}^{3}+3 G_{\eta_{u}, \eta_{u}}+6 G_{\eta_{u}, \eta_{d}}+3 G_{\eta_{d}, \eta_{d}}\right] \\
& +\frac{g_{A} g_{1}}{3}\left[2 m_{d d}^{3}-m_{u d}^{3}+m_{j d}^{3}+m_{l d}^{3}+3 G_{\eta_{d}, \eta_{d}}+3 G_{\eta_{u}, \eta_{d}}\right] \\
& \left.+\frac{g_{\Delta N}^{2}}{9 \pi}\left[5 F_{u d}+F_{d d}+F_{j d}+F_{l d}+2 F_{j u}+2 F_{l u}+2 E_{\eta_{d}, \eta_{d}}+2 E_{\eta_{u}, \eta_{u}}-4 E_{\eta_{u}, \eta_{d}}\right]\right)
\end{aligned}
$$

Our expressions for both the proton and neutron masses collapse down to those of isospinsymmetric QCD [18] in the limit $m_{j}, m_{l}, m_{u}, m_{d} \rightarrow \bar{m}$,

$$
M_{N}=M_{0}-2 \bar{m}\left(\alpha_{M}+2 \sigma_{M}\right)-\frac{1}{8 \pi f^{2}}\left[\frac{3}{2} g_{A}^{2} m_{\pi}^{3}+\frac{4 g_{\Delta N}^{2}}{3 \pi} F_{\pi}\right] .
$$

In obtaining this result we have used the fact that $G_{\eta_{d}, \eta_{d}} \rightarrow-\frac{1}{2} m_{\pi}^{3}$, and $E_{\eta_{u}, \eta_{u}} \rightarrow-\frac{1}{2} F_{\pi}$. By varying both the sea-quark and valence-quark masses over a suitable range and determining the proton and neutron masses, the constants $M_{0}, \alpha_{M}^{(P Q)}, \beta_{M}^{(P Q)}$ and $\sigma_{M}^{(P Q)}$ can, in principle, be determined for two-flavor QCD.

\section{NUCLEON MAGNETIC MOMENTS}

The nucleon magnetic moments have been computed on the lattice in both quenched and unquenched QCD using quark masses significantly larger than those of nature [19]. In order to define the magnetic moments of the nucleons in PQQCD, one must first define the light-quark electric-charge matrix. As discussed in detail in Ref. [8], the extension of the electric-charge matrix from QCD, where

$$
\mathcal{Q}=\operatorname{diag}\left(+\frac{2}{3},-\frac{1}{3}\right)
$$

to PQQCD is not unique, but is constrained by the requirement of recovering QCD in the limit that $m_{j} \rightarrow m_{u}$ and $m_{l} \rightarrow m_{d}$. The most general charge matrix whose matrix elements reduce down to those of QCD (keeping the valence-quark charges fixed) is

$$
\mathcal{Q}^{(P Q)}=\operatorname{diag}\left(+\frac{2}{3},-\frac{1}{3}, q_{j}, q_{l}, q_{j}, q_{l}\right) .
$$

For subsequent discussions we define $\mathcal{Q}_{\xi+}^{(P Q)}=\frac{1}{2}\left(\xi^{\dagger} \mathcal{Q}^{(P Q)} \xi+\xi \mathcal{Q}^{(P Q)} \xi^{\dagger}\right)$, and $\mathcal{Q}_{\xi+}=$ $\frac{1}{2}\left(\xi^{\dagger} \mathcal{Q} \xi+\xi \mathcal{Q} \xi^{\dagger}\right)$. 
Studying the behavior of the magnetic moments for a variety of charge-matrices with different relative contributions of the singlet and adjoint representations will yield the desired low-energy constants in the chiral Lagrangian [8,20]. The additional freedom introduced by the charges $q_{l}$ and $q_{j}$ is a blessing rather than a curse due to the fact that different extensions correspond to different weightings of disconnected diagrams in lattice simulations. Thus the impact of disconnected diagrams whose numerical evaluation, in some cases, converges slowly (and hence can induce large uncertainties) can be minimized by a suitable choice of $\mathcal{Q}^{(P Q)}$. Furthermore, in PQ $\chi \mathrm{PT}$ the contribution of one-loop diagrams involving the more massive sea-quarks can be minimized (order-by-order) by an appropriate choice of charges, thereby improving the convergence of the chiral expansion.

In QCD, there are two invariants that can be constructed at LO in the chiral expansion to describe the magnetic moments of the nucleons,

$$
\mathcal{L}=\frac{e}{4 M_{N}} F_{\mu \nu}\left(\mu_{A} \operatorname{Tr}\left[\mathcal{Q}_{\xi_{+}}\right] \bar{N} \sigma^{\mu \nu} N+\mu_{B} \bar{N} \sigma^{\mu \nu} \mathcal{Q}_{\xi_{+}} N\right)
$$

where $F_{\mu \nu}$ is the electromagnetic field-strength tensor, and $M_{N}$ is the physical value of the nucleon mass. More conventionally, this is written in terms of the isoscalar and isovector operators

$$
\mathcal{L}=\frac{e}{4 M_{N}} F_{\mu \nu}\left(\mu_{0} \bar{N} \sigma^{\mu \nu} N+\mu_{1} \bar{N} \sigma^{\mu \nu} \tau_{\xi+}^{3} N\right)
$$

where $\mu_{0}=\left(2 \mu_{A}+\mu_{B}\right) / 6$ is the isoscalar nucleon magnetic moment, $\mu_{1}=\mu_{B} / 2$ is the isovector nucleon magnetic moment, and $\tau_{\xi+}^{a}=\frac{1}{2}\left(\xi^{\dagger} \tau^{a} \xi+\xi \tau^{a} \xi^{\dagger}\right)$. In PQQCD, the LO Lagrange density contributing to the magnetic moments of the nucleons has the form

$$
\begin{aligned}
& \mathcal{L}=\frac{e}{4 M_{N}} F_{\mu \nu} {\left[\mu_{\alpha}\left(\overline{\mathcal{B}} \sigma^{\mu \nu} \mathcal{B} \mathcal{Q}_{\xi+}^{(P Q)}\right)+\mu_{\beta}\left(\overline{\mathcal{B}} \sigma^{\mu \nu} \mathcal{Q}_{\xi+}^{(P Q)} \mathcal{B}\right)\right.} \\
&\left.+\mu_{\gamma} \operatorname{str}\left[\mathcal{Q}_{\xi+}^{(P Q)}\right]\left(\overline{\mathcal{B}} \sigma^{\mu \nu} \mathcal{B}\right)\right]
\end{aligned}
$$

It is interesting to note that there is one more operator in PQQCD than there is in QCD at LO. The coefficients in the QCD and PQQCD LO Lagrange densities are related by

$$
\mu_{0}=\frac{1}{6}\left[\mu_{\alpha}+\mu_{\beta}+2 \mu_{\gamma}\right], \mu_{1}=\frac{1}{6}\left[2 \mu_{\alpha}-\mu_{\beta}\right] .
$$

At the order to which we will be working, $m_{q}^{1 / 2}$, it is convenient to write the proton and neutron magnetic moments in terms of the $\mu_{0,1}$. However, higher order calculations will need to be performed using $\mu_{\alpha, \beta, \gamma}$ directly.

Up to order $m_{q}^{1 / 2}$, it is convenient to write the magnetic moment of the $i$-th nucleon as

$$
\mu_{i}=\alpha_{i}+\frac{M_{N}}{4 \pi f^{2}}\left[\beta_{i}+\beta_{i}^{\prime}\right]+\ldots
$$

where the tree-level contributions, $\alpha_{i}$, are given by the Lagrange density in eq. (46). The $\beta$ and $\beta^{\prime}$ contributions arise from the one-loop diagrams shown in Fig. \&, involving nucleons in the $\mathbf{7 0}$ and resonances in the $\mathbf{4 4}$ dimensional representations, respectively. For the proton we find 

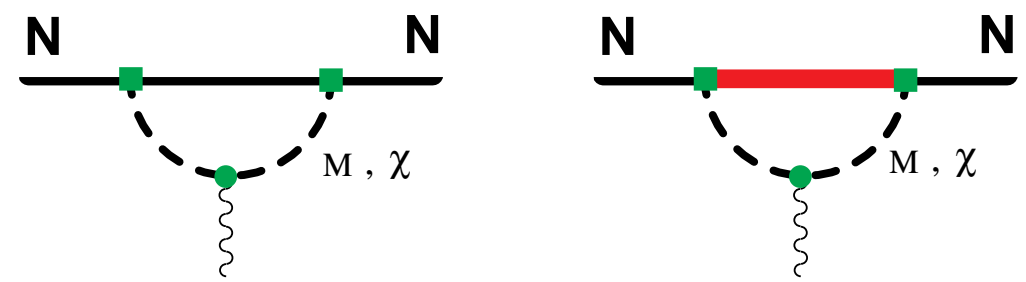

FIG. 2. One-loop graphs that give contributions of the form $\sim \sqrt{m_{Q}}$ to the magnetic moments of the nucleons. A solid, thick-solid and dashed line denote a 70-nucleon, 44-resonance, and a meson, respectively. The solid-squares denote an axial coupling from eq. (33), while the solid circle denotes a minimal coupling to the electromagnetic field.

$$
\begin{aligned}
\alpha_{p}= & \mu_{0}+\mu_{1} \\
\beta_{p}= & \frac{g_{A}^{2}}{9}\left[4 m_{u u}-5 m_{u d}-4 m_{j u}-4 m_{l u}\right]+\frac{2 g_{1} g_{A}}{9}\left[m_{u d}+m_{u u}-m_{j u}-m_{l u}\right] \\
& +\frac{g_{1}^{2}}{36}\left[m_{u d}+4 m_{u u}-3 m_{d d}+3 m_{j d}-4 m_{j u}+3 m_{l d}-4 m_{l u}\right] \\
& +q_{j}\left(\frac{2 g_{A}^{2}}{3}\left[m_{j u}-m_{u u}\right]+\frac{g_{1} g_{A}}{3}\left[m_{j u}-m_{u u}\right]+\frac{g_{1}^{2}}{6}\left[m_{j u}-m_{u u}+\frac{3}{2} m_{j d}-\frac{3}{2} m_{u d}\right]\right) \\
& +q_{l}\left(\frac{2 g_{A}^{2}}{3}\left[m_{l u}-m_{u d}\right]+\frac{g_{1} g_{A}}{3}\left[m_{l u}-m_{u d}\right]+\frac{g_{1}^{2}}{6}\left[m_{l u}-m_{u d}+\frac{3}{2} m_{l d}-\frac{3}{2} m_{d d}\right]\right) \\
\beta_{p}^{\prime}= & g_{\Delta N}^{2} \frac{1}{27}\left[\mathcal{F}_{d d}-\mathcal{F}_{u u}-6 \mathcal{F}_{u d}-\mathcal{F}_{j d}+\mathcal{F}_{j u}-\mathcal{F}_{l d}+\mathcal{F}_{l u}\right. \\
& \left.+\frac{3}{2} q_{j}\left(\mathcal{F}_{u u}+2 \mathcal{F}_{u d}-\mathcal{F}_{j u}-2 \mathcal{F}_{j d}\right)+\frac{3}{2} q_{l}\left(\mathcal{F}_{u d}+2 \mathcal{F}_{d d}-\mathcal{F}_{l u}-2 \mathcal{F}_{l d}\right)\right],
\end{aligned}
$$

where the function $\mathcal{F}_{i}=\mathcal{F}\left(m_{i}, \Delta, \mu\right)$ is

$$
\pi \mathcal{F}(m, \Delta, \mu)=\sqrt{\Delta^{2}-m^{2}} \log \left(\frac{\Delta-\sqrt{\Delta^{2}-m^{2}+i \epsilon}}{\Delta+\sqrt{\Delta^{2}-m^{2}+i \epsilon}}\right)-\Delta \log \left(\frac{m^{2}}{\mu^{2}}\right) .
$$

In the limit $\Delta \rightarrow 0, \mathcal{F}(m, 0, \mu)=m$. For the neutron we find

$$
\begin{aligned}
\alpha_{n}= & \mu_{0}-\mu_{1} \\
\beta_{n}= & \frac{g_{A}^{2}}{9}\left[7 m_{u d}+2 m_{l d}+2 m_{j d}-2 m_{d d}\right]+\frac{g_{1} g_{A}}{9}\left[m_{j d}+m_{l d}-m_{u d}-m_{d d}\right] \\
& +\frac{g_{1}^{2}}{18}\left[3 m_{u u}+2 m_{u d}-m_{d d}+m_{j d}-3 m_{j u}+m_{l d}-3 m_{l u}\right] \\
& +q_{j}\left(\frac{2 g_{A}^{2}}{3}\left[m_{j d}-m_{u d}\right]+\frac{g_{1} g_{A}}{3}\left[m_{j d}-m_{u d}\right]+\frac{g_{1}^{2}}{6}\left[m_{j d}-m_{u d}+\frac{3}{2} m_{j u}-\frac{3}{2} m_{u u}\right]\right) \\
& +q_{l}\left(\frac{2 g_{A}^{2}}{3}\left[m_{l d}-m_{d d}\right]+\frac{g_{1} g_{A}}{3}\left[m_{l d}-m_{d d}\right]+\frac{g_{1}^{2}}{6}\left[m_{l d}-m_{d d}+\frac{3}{2} m_{l u}-\frac{3}{2} m_{u d}\right]\right) \\
\beta_{n}^{\prime}= & g_{\Delta N}^{2} \frac{1}{54}\left[\mathcal{F}_{d d}-4 \mathcal{F}_{u u}+9 \mathcal{F}_{u d}-\mathcal{F}_{j d}+4 \mathcal{F}_{j u}-\mathcal{F}_{l d}+4 \mathcal{F}_{l u}\right. \\
& \left.+3 q_{j}\left(\mathcal{F}_{u d}+2 \mathcal{F}_{u u}-\mathcal{F}_{j d}-2 \mathcal{F}_{j u}\right)+3 q_{l}\left(\mathcal{F}_{d d}+2 \mathcal{F}_{u d}-\mathcal{F}_{l d}-2 \mathcal{F}_{l u}\right)\right] .
\end{aligned}
$$


These expressions reduce to their QCD counterparts 21 23.

$$
\begin{aligned}
& \mu_{p}=\mu_{0}+\mu_{1}-\frac{M_{N}}{4 \pi f^{2}}\left[g_{A}^{2} m_{\pi^{+}}+\frac{2}{9} g_{\Delta N}^{2} \mathcal{F}_{\pi^{+}}\right] \\
& \mu_{n}=\mu_{0}-\mu_{1}+\frac{M_{N}}{4 \pi f^{2}}\left[g_{A}^{2} m_{\pi^{+}}+\frac{2}{9} g_{\Delta N}^{2} \mathcal{F}_{\pi^{+}}\right],
\end{aligned}
$$

when $m_{j} \rightarrow m_{u}$ and $m_{l} \rightarrow m_{d}$. Note that the results are independent of the charges $q_{j, l}$.

\section{MATRIX ELEMENTS OF ISOVECTOR TWIST-2 OPERATORS}

The forward matrix elements of twist-2 operators play an important role in hadronic structure as they are directly related to the moments of the parton distribution functions. There exist both quenched and unquenched lattice simulations of these matrix elements [24]. Recently, it was realized that the long-distance contributions to these matrix elements could be computed order-by-order in the chiral expansion using chiral perturbation theory [25 27]. These corrections have been applied to results from both quenched and unquenched lattice data [28], with interesting results. In addition, the long-distance contributions arising in QQCD and the large- $N_{c}$ limit of QCD have been computed in Ref. [29] and Ref. [30], respectively. Further, this technique has been applied to the off-forward matrix elements of twist-2 operators in order to study the spin structure of the proton [31]. In Ref. [8], the matrix elements of the isovector twist-2 operators were computed at the one-loop level in $S U(6 \mid 3)_{L} \otimes S U(6 \mid 3)_{R}$ PQ $\chi \mathrm{PT}$. Like the magnetic moments, the extension of the twist-2 matrix elements from QCD to PQQCD introduces a non-unique isovector charge matrix, which can be exploited to optimize the numerical simulations and the chiral expansion [8].

In QCD, the nonsinglet operators have the form,

$$
\mathcal{O}_{\mu_{1} \mu_{2} \ldots \mu_{n}}^{(n), a}=\frac{1}{n !} \bar{q} \tau^{a} \gamma_{\left\{\mu_{1}\right.}\left(i \stackrel{\leftrightarrow}{D}_{\mu_{2}}\right) \ldots\left(i \stackrel{\leftrightarrow}{D}_{\left.\mu_{n}\right\}}\right) q-\text { traces }
$$

where the $\{\ldots\}$ denotes symmetrization with respect to all Lorentz indices, and $\tau^{a}$ are Pauli matrices acting in flavor-space. They transform as $(\mathbf{3}, \mathbf{1}) \oplus(\mathbf{1}, \mathbf{3})$ under $S U(2)_{L} \otimes S U(2)_{R}$ chiral transformations [25,26]. Of particular interest to us are the isovector operators where $\tau^{3}=\operatorname{diag}(1,-1)$. At LO in the chiral expansion the $\mathcal{O}_{\mu_{1} \mu_{2} \ldots \mu_{n}}^{(n), 3}$ match onto [25] 27]

$$
\begin{aligned}
\mathcal{O}_{\mu_{1} \mu_{2} \ldots \mu_{n}}^{(n), 3} & \rightarrow a^{(n)}(i)^{n} \frac{f^{2}}{4}\left(\frac{1}{\Lambda_{\chi}}\right)^{n-1} \operatorname{Tr}\left[\Sigma^{\dagger} \tau^{3} \vec{\partial}_{\mu_{1}} \vec{\partial}_{\mu_{2}} \ldots \vec{\partial}_{\mu_{n}} \Sigma+\Sigma \tau^{3} \vec{\partial}_{\mu_{1}} \vec{\partial}_{\mu_{2}} \ldots \vec{\partial}_{\mu_{n}} \Sigma^{\dagger}\right] \\
+ & A^{(n)} v_{\mu_{1}} v_{\mu_{2}} \ldots v_{\mu_{n}} \bar{N} \tau_{\xi+}^{3} N \\
+ & \gamma^{(n)} v_{\mu_{1}} v_{\mu_{2}} \ldots v_{\mu_{n}} \bar{T}^{\alpha} \tau_{\xi+}^{3} T_{\alpha}+\sigma^{(n)} \frac{1}{n !} v_{\left\{\mu_{1}\right.} v_{\mu_{2}} \ldots v_{\mu_{n-2}} \bar{T}_{\mu_{n-1}} \tau_{\xi+}^{3} T_{\left.\mu_{n}\right\}} \\
& \quad-\text { traces }
\end{aligned}
$$

In PQQCD, the nonsinglet operators have the form

$$
{ }^{P Q} \mathcal{O}_{\mu_{1} \mu_{2} \ldots \mu_{n}}^{(n), a}=\frac{1}{n !} \bar{Q} \bar{\tau}^{a} \gamma_{\left\{\mu_{1}\right.}\left(i \stackrel{\leftrightarrow}{D}_{\mu_{2}}\right) \ldots\left(i \stackrel{\leftrightarrow}{D}{ }_{\left.\mu_{n}\right\}}\right) Q-\text { traces }
$$


where the $\bar{\tau}^{a}$ are super Pauli matrices, an extension of the Pauli matrices from two-flavor QCD to PQQCD. With the requirements that $\bar{\tau}^{3}$ is supertraceless and that the QCD matrix elements are recovered in the limit $m_{j} \rightarrow m_{u}, m_{l} \rightarrow m_{d}$, the most general flavor structure for $\bar{\tau}^{3}$ is (keeping the valence-quark charges fixed)

$$
\bar{\tau}^{3}=\left(1,-1, y_{j}, y_{l}, y_{j}, y_{l}\right) .
$$

For an arbitrary choice of the $y_{i}$, this operator contains both isovector and isoscalar components. It is purely isovector only when $y_{j}+y_{l}=0$. As a result, for arbitrary $y_{i}$, the usual isovector relations between matrix elements do not hold. The fact that disconnected diagrams can only be isoscalar renders this result obvious. At LO in the chiral expansion, matrix elements of the isovector operator ${ }^{P Q} \mathcal{O}_{\mu_{1} \mu_{2} \ldots \mu_{n}}^{(n), 3}$ are reproduced by operators of the form $8,25,27$

$$
\begin{aligned}
{ }^{P Q} \mathcal{O}_{\mu_{1} \mu_{2} \ldots \mu_{n}}^{(n), 3} & \rightarrow a^{(n)}(i)^{n} \frac{f^{2}}{4}\left(\frac{1}{\Lambda_{\chi}}\right)^{n-1} \operatorname{str}\left[\Sigma^{\dagger} \bar{\tau}^{3} \vec{\partial}_{\mu_{1}} \vec{\partial}_{\mu_{2}} \ldots \vec{\partial}_{\mu_{n}} \Sigma+\Sigma \bar{\tau}^{3} \vec{\partial}_{\mu_{1}} \vec{\partial}_{\mu_{2}} \ldots \vec{\partial}_{\mu_{n}} \Sigma^{\dagger}\right] \\
+ & \alpha^{(n)} v_{\mu_{1}} v_{\mu_{2}} \ldots v_{\mu_{n}}\left(\overline{\mathcal{B}} \mathcal{B} \bar{\tau}_{\xi+}^{3}\right)+\beta^{(n)} v_{\mu_{1}} v_{\mu_{2} \ldots v_{\mu_{n}}}\left(\overline{\mathcal{B}} \bar{\tau}_{\xi+}^{3} \mathcal{B}\right) \\
+ & \gamma^{(n)} v_{\mu_{1}} v_{\mu_{2}} \ldots v_{\mu_{n}}\left(\overline{\mathcal{T}}^{\alpha} \bar{\tau}_{\xi+}^{3} \mathcal{T}_{\alpha}\right)+\sigma^{(n)} \frac{1}{n !} v_{\left\{\mu_{1}\right.} v_{\mu_{2}} \ldots v_{\mu_{n-2}}\left(\overline{\mathcal{T}}_{\mu_{n-1}} \bar{\tau}_{\xi+}^{3} \mathcal{T}_{\left.\mu_{n}\right\}}\right) \\
& - \text { traces }
\end{aligned}
$$

where $\bar{\tau}_{\xi+}^{3}=\frac{1}{2}\left(\xi \bar{\tau}^{3} \xi^{\dagger}+\xi^{\dagger} \bar{\tau}^{3} \xi\right)$. In general, the coefficients $a^{(n)}, \alpha^{(n)}, \beta^{(n)}, \gamma^{(n)}$ and $\sigma^{(n)}$ are not constrained by symmetries and must be determined from elsewhere. However for $n=1$ they are fixed by the isospin charge of the hadrons to be

$$
a^{(1)}=+1 \quad, \quad \alpha^{(1)}=+2 \quad, \quad \beta^{(1)}=+1 \quad, \quad \gamma^{(1)}=-3 \quad, \quad \sigma^{(1)}=0 .
$$

Comparing the operators in the QCD and PQQCD chiral Lagrangians, one finds that the coefficients $\alpha^{(n)}$ and $\beta^{(n)}$ both contribute to $A^{(n)}$ in the QCD limit of PQQCD, but away from this limit the operators are independent.

At the one-loop level there are contributions from counterterms involving one insertion of the quark mass matrix $m_{Q}$,

$$
\begin{aligned}
P Q \mathcal{O}_{\mu_{1} \mu_{2} \ldots \mu_{n}}^{(n), 3} & \rightarrow\left[b_{1}^{(n)} \overline{\mathcal{B}}^{k j i}\left\{\bar{\tau}_{\xi+}^{3}, \mathcal{M}_{+}\right\}_{i}^{n} \mathcal{B}_{n j k}\right. \\
& +b_{2}^{(n)}(-)^{\left(\eta_{i}+\eta_{j}\right)\left(\eta_{k}+\eta_{n}\right)} \overline{\mathcal{B}}^{k j i}\left\{\bar{\tau}_{\xi+}^{3}, \mathcal{M}_{+}\right\}_{k}^{n} \mathcal{B}_{i j n} \\
& +b_{3}^{(n)}(-)^{\eta_{l}\left(\eta_{j}+\eta_{n}\right)} \overline{\mathcal{B}}^{k j i}\left(\bar{\tau}_{\xi+}^{3}\right)_{i}^{l}\left(\mathcal{M}_{+}\right)_{j}^{n} \mathcal{B}_{l n k} \\
& +b_{4}^{(n)}(-)^{\eta_{l} \eta_{j}+1} \overline{\mathcal{B}}^{k j i}\left(\left(\bar{\tau}_{\xi+}^{3}\right)_{i}^{l}\left(\mathcal{M}_{+}\right)_{j}^{n}+\left(\mathcal{M}_{+}\right)_{i}^{l}\left(\bar{\tau}^{3}\right)_{j}^{n}\right) \mathcal{B}_{n l k} \\
& +b_{5}^{(n)}(-)^{\eta_{i}\left(\eta_{l}+\eta_{j}\right)} \overline{\mathcal{B}}^{k j i}\left(\bar{\tau}_{\xi+}^{3}\right)_{j}^{l}\left(\mathcal{M}_{+}\right)_{i}^{n} \mathcal{B}_{n l k}+b_{6}^{(n)} \overline{\mathcal{B}}^{k j i}\left(\bar{\tau}_{\xi+}^{3}\right)_{i}^{l} \mathcal{B}_{l j k} \operatorname{str}\left(\mathcal{M}_{+}\right) \\
& +b_{7}^{(n)}(-)^{\left(\eta_{i}+\eta_{j}\right)\left(\eta_{k}+\eta_{n}\right)} \overline{\mathcal{B}}^{k j i}\left(\bar{\tau}_{\xi+}^{3}\right)_{k}^{n} \mathcal{B}_{i j n} \operatorname{str}\left(\mathcal{M}_{+}\right) \\
& \left.+b_{8}^{(n)} \overline{\mathcal{B}}^{k j i} \mathcal{B}_{i j k} \operatorname{str}\left(\bar{\tau}_{\xi+}^{3} \mathcal{M}_{+}\right)\right] v_{\mu_{1}} v_{\mu_{2} \ldots v_{\mu_{n}}}-\operatorname{traces}
\end{aligned}
$$

where the coefficients $b_{1}^{(n)}, \ldots b_{8}^{(n)}$ are to be determined. The only constraint that exists on the $b_{i}^{(n)}$ is that the isospin charge of each nucleon is absolutely normalized, and thus $b_{i}^{(1)}=0$. 
The forward matrix elements of ${ }^{P Q} \mathcal{O}_{\mu_{1} \mu_{2} \ldots \mu_{n}}^{(n), 3}$ in the $i$-th nucleon at the one-loop level can be written as

$$
\begin{array}{r}
\left\langle{ }^{P Q} \mathcal{O}_{\mu_{1} \mu_{2} \ldots \mu_{n}}^{(n), 3}\right\rangle_{i}=\left[\rho_{i}^{(n)}+\frac{1-\delta^{n 1}}{16 \pi^{2} f^{2}}\left(\eta_{i}^{(n), 0}-\rho_{i}^{(n)} w_{i}+y_{j} \eta_{i}^{(n), j}+y_{l} \eta_{i}^{(n), l}\right)\right. \\
\left.c_{i}^{(n), 0}+y_{j} c_{i}^{(n), j}+y_{l} c_{i}^{(n), l}\right] \bar{U}_{i} v_{\mu_{1}} v_{\mu_{2}} \ldots v_{\mu_{n}} U_{i}-\text { traces }
\end{array}
$$

where $\rho_{i}^{(n)}$ are the LO contributions. The factor of $1-\delta^{n 1}$ appears in the higher order corrections because the isovector charge is not renormalized. The diagrams shown in Fig. 3 give the leading non-analytic contributions to the wavefunction renormalization, $w_{i}$, the vertex contributions, $\eta_{i}^{(n), 0}$, that are independent of the charges of the ghost- and sea-quarks, and to the vertex contributions, $\eta_{i}^{(n), j, l}$, associated with the charges of the ghost- and valencequarks. For the proton we find

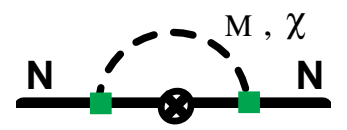

(a)

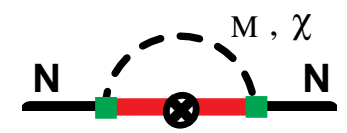

(c)

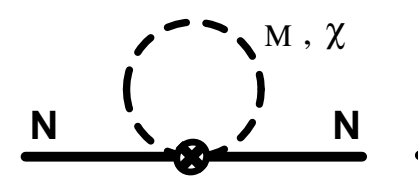

(e)

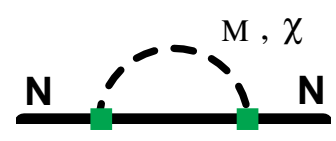

(g)

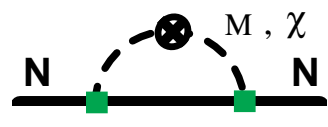

(b)

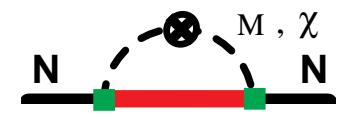

(d)

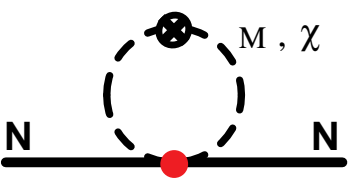

(f)

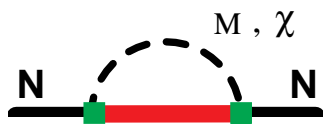

(h)

FIG. 3. One-loop graphs that give contributions of the form $\sim m_{Q} \log m_{Q}$ to the matrix elements of the isovector twist-2 operators in the nucleon. A solid, thick-solid and dashed line denote a 70-nucleon, 44-resonance, and a meson, respectively. The solid-squares denote an axial coupling given in eq.(33), while the solid circle denotes an insertion of the strong two-pion-nucleon interaction given in eq.(20). The crossed circle denotes an insertion of the tree-level matrix element of ${ }^{P Q} \mathcal{O}_{\mu_{1} \mu_{2}}^{(n), a \mu_{n}}$. Diagrams (a) to (f) are vertex corrections, while diagrams ( $g$ ) and ( $h$ ) give rise to wavefunction renormalization.

$$
\rho_{p}^{(n)}=\frac{1}{3}\left(2 \alpha^{(n)}-\beta^{(n)}\right)
$$




$$
\begin{aligned}
& w_{p}=g_{A}^{2}\left(L_{u d}+L_{u u}+2 L_{j u}+2 L_{l u}+3 R_{\eta_{u}, \eta_{u}}\right) \\
& +g_{1} g_{A}\left(2 L_{u u}-L_{u d}+L_{j u}+L_{l u}+3 R_{\eta_{u}, \eta_{u}}+3 R_{\eta_{u}, \eta_{d}}\right) \\
& +\frac{g_{1}^{2}}{4}\left(L_{u u}-5 L_{u d}+2 L_{l u}+3 L_{l d}+2 L_{j u}+3 L_{j d}+3 R_{\eta_{u}, \eta_{u}}+6 R_{\eta_{u}, \eta_{d}}+3 R_{\eta_{d}, \eta_{d}}\right) \\
& +\frac{1}{3} g_{\Delta N}^{2}\left(5 J_{u d}+J_{u u}+J_{j u}+J_{l u}+2 J_{j d}+2 J_{l d}+2 \mathcal{T}_{\eta_{u}, \eta_{u}}+2 \mathcal{T}_{\eta_{d}, \eta_{d}}-4 \mathcal{T}_{\eta_{u}, \eta_{d}}\right) \\
& \eta_{p}^{(n), 0}=3 g_{A}^{2} \rho_{p}^{(n)}\left(L_{u u}-L_{u d}+R_{\eta_{u}, \eta_{u}}\right) \\
& +\frac{g_{1} g_{A}}{2}\left[\alpha^{(n)}\left(3 L_{u u}-L_{u d}+L_{j u}+L_{l u}+4 R_{\eta_{u}, \eta_{d}}+4 R_{\eta_{u}, \eta_{u}}\right)\right. \\
& \left.+2 \beta^{(n)}\left(L_{u d}-L_{j u}-L_{l u}-R_{\eta_{u}, \eta_{d}}-R_{\eta_{u}, \eta_{u}}\right)\right] \\
& +\frac{g_{1}^{2}}{8}\left[\alpha ^ { ( n ) } \left(3 L_{u u}-6 L_{u d}-L_{d d}+5 L_{j d}+L_{j u}+5 L_{l d}+L_{l u}\right.\right. \\
& \left.+4 R_{\eta_{u}, \eta_{u}}+8 R_{\eta_{u}, \eta_{d}}+4 R_{\eta_{d}, \eta_{d}}\right) \\
& \left.+2 \beta^{(n)}\left(L_{j d}-L_{j u}+L_{l d}-L_{l u}-2 L_{d d}-R_{\eta_{u}, \eta_{u}}-2 R_{\eta_{u}, \eta_{d}}-R_{\eta_{d}, \eta_{d}}\right)\right] \\
& +\frac{2}{9} g_{\Delta N}^{2}\left(\gamma^{(n)}-\frac{\sigma^{(n)}}{3}\right)\left(J_{d d}-J_{u u}-6 J_{u d}-2 J_{j d}-2 J_{l d}-\mathcal{T}_{\eta_{u}, \eta_{u}}-\mathcal{T}_{\eta_{d}, \eta_{d}}+2 \mathcal{T}_{\eta_{u}, \eta_{d}}\right) \\
& +\frac{1}{6} \alpha^{(n)}\left(5 L_{u u}-4 L_{u d}-L_{d d}-5 L_{j u}-5 L_{l u}+L_{j d}+L_{l d}\right) \\
& +\frac{1}{3} \beta^{(n)}\left(L_{u u}+L_{u d}-2 L_{d d}-L_{j u}-L_{l u}+2 L_{j d}+2 L_{l d}\right) \\
& \eta_{p}^{(n), j}=g_{A}^{2} \alpha^{(n)}\left(L_{j u}-L_{u u}\right)+\frac{g_{1} g_{A}}{2} \alpha^{(n)}\left(L_{j u}-L_{u u}\right) \\
& +\frac{g_{1}^{2}}{8}\left[\alpha^{(n)}\left(L_{j d}+L_{j u}-L_{u d}-L_{u u}\right)+\beta^{(n)}\left(4 L_{j d}+2 L_{j u}-4 L_{u d}-2 L_{u u}\right)\right] \\
& +\frac{1}{9} g_{\Delta N}^{2}\left(\gamma^{(n)}-\frac{\sigma^{(n)}}{3}\right)\left(J_{u u}+2 J_{u d}-2 J_{j d}-J_{j u}\right) \\
& +\frac{1}{6} \alpha^{(n)}\left(-5 L_{u u}-L_{u d}+5 L_{j u}+L_{j d}\right)+\frac{1}{3} \beta^{(n)}\left(-L_{u u}-2 L_{u d}+L_{j u}+2 L_{j d}\right) \\
& \eta_{p}^{(n), l}=g_{A}^{2} \alpha^{(n)}\left(L_{l u}-L_{u d}\right)+\frac{g_{1} g_{A}}{2} \alpha^{(n)}\left(L_{l u}-L_{u d}\right) \\
& +\frac{g_{1}^{2}}{8}\left[\alpha^{(n)}\left(L_{l d}+L_{l u}-L_{u d}-L_{d d}\right)+\beta^{(n)}\left(4 L_{l d}+2 L_{l u}-4 L_{d d}-2 L_{u d}\right)\right] \\
& +\frac{1}{9} g_{\Delta N}^{2}\left(\gamma^{(n)}-\frac{\sigma^{(n)}}{3}\right)\left(J_{u d}+2 J_{d d}-2 J_{l d}-J_{l u}\right) \\
& +\frac{1}{6} \alpha^{(n)}\left(-5 L_{u d}-L_{d d}+5 L_{l u}+L_{l d}\right)+\frac{1}{3} \beta^{(n)}\left(-2 L_{d d}-L_{u d}+L_{l u}+2 L_{l d}\right) \\
& c_{p}^{(n), 0}=\frac{1}{3} m_{u}\left(2 b_{1}^{(n)}+5 b_{2}^{(n)}-\frac{3}{2} b_{3}^{(n)}+3 b_{4}^{(n)}+3 b_{8}^{(n)}\right) \\
& +\frac{1}{3} m_{d}\left(-4 b_{1}^{(n)}-b_{2}^{(n)}+\frac{1}{2} b_{3}^{(n)}-2 b_{4}^{(n)}+2 b_{5}^{(n)}-3 b_{8}^{(n)}\right) \\
& +\frac{1}{3}\left(m_{j}+m_{l}\right)\left(-b_{6}^{(n)}+2 b_{7}^{(n)}\right) \\
& c_{p}^{(n), j}=\left(m_{j}-m_{u}\right) b_{8}^{(n)}
\end{aligned}
$$




$$
c_{p}^{(n), l}=\left(m_{l}-m_{d}\right) b_{8}^{(n)},
$$

where, for the contributions from the $\mathbf{7 0}$ intermediate states, we have defined $L_{a b}=$ $m_{a b}^{2} \log \left(m_{a b}^{2} / \mu^{2}\right)$, and $R_{x, y}=\mathcal{H}\left(L_{x}, L_{y}, L_{X}\right)$. For the contributions from the 44 intermediate states, we have defined $J_{a b}=J\left(m_{a b}, \Delta, \mu\right)$, with

$$
J(m, \Delta, \mu)=\left(m^{2}-2 \Delta^{2}\right) \log \left(\frac{m^{2}}{\mu^{2}}\right)+2 \Delta \sqrt{\Delta^{2}-m^{2}} \log \left(\frac{\Delta-\sqrt{\Delta^{2}-m^{2}+i \epsilon}}{\Delta+\sqrt{\Delta^{2}-m^{2}+i \epsilon}}\right)
$$

and $\mathcal{T}_{x, y}=\mathcal{H}\left(J_{x}, J_{y}, J_{X}\right)$. For the neutron we find

$$
\begin{aligned}
& \rho_{n}^{(n)}=-\frac{1}{3}\left(2 \alpha^{(n)}-\beta^{(n)}\right) \\
& w_{n}=g_{A}^{2}\left(L_{d d}+L_{u d}+2 L_{j d}+2 L_{l d}+3 R_{\eta_{d}, \eta_{d}}\right) \\
& +g_{1} g_{A}\left(2 L_{d d}-L_{u d}+L_{j d}+L_{l d}+3 R_{\eta_{u}, \eta_{d}}+3 R_{\eta_{d}, \eta_{d}}\right) \\
& +\frac{g_{1}^{2}}{4}\left(L_{d d}-5 L_{u d}+2 L_{j d}+3 L_{j u}+2 L_{l d}+3 L_{l u}+3 R_{\eta_{u}, \eta_{u}}+6 R_{\eta_{u}, \eta_{d}}+3 R_{\eta_{d}, \eta_{d}}\right) \\
& +\frac{1}{3} g_{\Delta N}^{2}\left(5 J_{u d}+J_{d d}+2 J_{j u}+2 J_{l u}+J_{j d}+J_{l d}+2 \mathcal{T}_{\eta_{u}, \eta_{u}}+2 \mathcal{T}_{\eta_{d}, \eta_{d}}-4 \mathcal{T}_{\eta_{u}, \eta_{d}}\right) \\
& \eta_{n}^{(n), 0}=3 g_{A}^{2} \rho_{p}^{(n)}\left(L_{d d}-L_{u d}+R_{\eta_{d}, \eta_{d}}\right) \\
& +\frac{g_{1} g_{A}}{2}\left[\alpha^{(n)}\left(L_{u d}-3 L_{d d}-L_{j d}-L_{l d}-4 R_{\eta_{u}, \eta_{d}}-4 R_{\eta_{d}, \eta_{d}}\right)\right. \\
& \left.+2 \beta^{(n)}\left(L_{j d}+L_{l d}-L_{u d}+R_{\eta_{u}, \eta_{d}}+R_{\eta_{d}, \eta_{d}}\right)\right] \\
& +\frac{g_{1}^{2}}{8}\left[\alpha ^ { ( n ) } \left(6 L_{u d}+L_{u u}-3 L_{d d}-L_{j d}-5 L_{j u}-L_{l d}-5 L_{l u}\right.\right. \\
& \left.-4 R_{\eta_{u}, \eta_{u}}-8 R_{\eta_{u}, \eta_{d}}-4 R_{\eta_{d}, \eta_{d}}\right) \\
& \left.+2 \beta^{(n)}\left(2 L_{u u}+L_{j d}-L_{j u}+L_{l d}-L_{l u}+R_{\eta_{u}, \eta_{u}}+2 R_{\eta_{u}, \eta_{d}}+R_{\eta_{d}, \eta_{d}}\right)\right] \\
& +\frac{2}{9} g_{\Delta N}^{2}\left(\gamma^{(n)}-\frac{\sigma^{(n)}}{3}\right)\left(J_{d d}-J_{u u}+6 J_{u d}+2 J_{j u}+2 J_{l u}+\mathcal{T}_{\eta_{u}, \eta_{u}}+\mathcal{T}_{\eta_{d}, \eta_{d}}-2 \mathcal{T}_{\eta_{u}, \eta_{d}}\right) \\
& +\frac{1}{6} \alpha^{(n)}\left(-5 L_{d d}+4 L_{u d}+L_{u u}+5 L_{j d}+5 L_{l d}-L_{j u}-L_{l u}\right) \\
& +\frac{1}{3} \beta^{(n)}\left(-L_{d d}-L_{u d}+2 L_{u u}-2 L_{j u}-2 L_{l u}+L_{j d}+L_{l d}\right) \\
& \eta_{n}^{(n), j}=g_{A}^{2} \alpha^{(n)}\left(L_{j d}-L_{u d}\right)+\frac{g_{1} g_{A}}{2} \alpha^{(n)}\left(L_{j d}-L_{u d}\right) \\
& +\frac{g_{1}^{2}}{8}\left[\alpha^{(n)}\left(L_{j d}+L_{j u}-L_{u d}-L_{u u}\right)+2 \beta^{(n)}\left(L_{j d}+2 L_{j u}-L_{u d}-2 L_{u u}\right)\right] \\
& +\frac{1}{9} g_{\Delta N}^{2}\left(\gamma^{(n)}-\frac{\sigma^{(n)}}{3}\right)\left(J_{u d}+2 J_{u u}-2 J_{j u}-J_{j d}\right) \\
& +\frac{1}{6} \alpha^{(n)}\left(-5 L_{u d}-L_{u u}+5 L_{j d}+L_{j u}\right)+\frac{1}{3} \beta^{(n)}\left(-L_{u d}-2 L_{u u}+L_{j d}+2 L_{j u}\right) \\
& \eta_{n}^{(n), l}=g_{A}^{2} \alpha^{(n)}\left(L_{l d}-L_{d d}\right)+\frac{g_{1} g_{A}}{2} \alpha^{(n)}\left(L_{l d}-L_{d d}\right) \\
& +\frac{g_{1}^{2}}{8}\left[\alpha^{(n)}\left(L_{l d}+L_{l u}-L_{d d}-L_{u d}\right)+2 \beta^{(n)}\left(L_{l d}+2 L_{l u}-L_{d d}-2 L_{u d}\right)\right]
\end{aligned}
$$




$$
\begin{aligned}
& +\frac{1}{9} g_{\Delta N}^{2}\left(\gamma^{(n)}-\frac{\sigma^{(n)}}{3}\right)\left(J_{d d}+2 J_{u d}-2 J_{l u}-J_{l d}\right) \\
& +\frac{1}{6} \alpha^{(n)}\left(-5 L_{d d}-L_{u d}+5 L_{l d}+L_{l u}\right)+\frac{1}{3} \beta^{(n)}\left(-2 L_{u d}-L_{d d}+L_{l d}+2 L_{l u}\right) \\
c_{n}^{(n), 0}= & \frac{1}{3} m_{u}\left(4 b_{1}^{(n)}+b_{2}^{(n)}-\frac{1}{2} b_{3}^{(n)}+2 b_{4}^{(n)}-2 b_{5}^{(n)}+3 b_{8}^{(n)}\right) \\
& +\frac{1}{3} m_{d}\left(-2 b_{1}^{(n)}-5 b_{2}^{(n)}+\frac{3}{2} b_{3}^{(n)}-3 b_{4}^{(n)}-3 b_{8}^{(n)}\right) \\
& +\frac{1}{3}\left(m_{j}+m_{l}\right)\left(b_{6}^{(n)}-2 b_{7}^{(n)}\right) \\
c_{n}^{(n), j}= & \left(m_{j}-m_{u}\right) b_{8}^{(n)} \\
c_{n}^{(n), l}= & \left(m_{l}-m_{d}\right) b_{8}^{(n)} .
\end{aligned}
$$

In the QCD and isospin limits, these expressions reduce down to those obtained in Refs. 25,26],

$$
\begin{aligned}
\left\langle\mathcal{O}_{\mu_{1} \mu_{2} \ldots \mu_{n}}^{(n), 3}\right\rangle_{p}=\bar{U}_{p} v_{\mu_{1}} v_{\mu_{2} \ldots} \ldots v_{\mu_{n}} U_{p}\left[\rho_{p}^{(n)}\left(1-\frac{\left(3 g_{A}^{2}+1\right)\left(1-\delta^{n 1}\right)}{8 \pi^{2} f^{2}} L_{\pi}\right)\right. & \\
- & \left.\frac{g_{\Delta N}^{2}\left(1-\delta^{n 1}\right)}{4 \pi^{2} f^{2}} J_{\pi}\left[\rho_{p}^{(n)}+\frac{5}{9} \gamma^{(n)}-\frac{5}{27} \sigma^{(n)}\right]\right]- \text { traces }
\end{aligned}
$$

where we have not shown the contribution from local counterterms involving a single insertion of $m_{q}$. Notice that the QCD limit is independent of the coupling $g_{1}$, as required. Furthermore, in the isospin limit alone, where $m_{j}=m_{l}$ and $m_{u}=m_{d}$, these expressions reduce down to those obtained in three-flavor PQQCD [8] when the strange quark mass is taken to be very heavy.

\section{THE AXIAL-VECTOR CURRENT}

Matrix elements of the axial-vector current, $\bar{q} \tau^{a} \gamma_{\mu} \gamma_{5} q$, are extensively studied on the lattice [24]. In $\chi \mathrm{PT}$, there have been numerous computations of these matrix elements [14, 32 at the one-loop level, both including and excluding the decuplet as an intermediate state. In analogy to the extension of the electric- and isovector-charge matrices to PQQCD, there is a non-uniqueness in the extension of the isovector axial currents, $\bar{Q} \bar{\tau}^{a} \gamma_{\mu} \gamma_{5} Q$, to PQQCD. We use the charge matrix in eq. (56) for the flavor-conserving currents, and for the flavorchanging current we replace $\tau^{3}$ with $\tau^{+}$in the upper $2 \times 2$ block. At LO in the chiral expansion, the axial current takes the form

$$
\begin{aligned}
{ }^{(P Q)} j_{\mu, 5}^{3} & \rightarrow 2 \alpha\left(\overline{\mathcal{B}} S_{\mu} \mathcal{B} \bar{\tau}_{\xi+}^{3}\right)+2 \beta\left(\overline{\mathcal{B}} S_{\mu} \bar{\tau}_{\xi+}^{3} \mathcal{B}\right)+2 \mathcal{H}\left(\overline{\mathcal{T}}^{\nu} S_{\mu} \bar{\tau}_{\xi+}^{3} \mathcal{T}_{\nu}\right) \\
& +\sqrt{\frac{3}{2}} \mathcal{C}\left[\left(\overline{\mathcal{T}}_{\mu} \bar{\tau}_{\xi+}^{3} \mathcal{B}\right)+\left(\overline{\mathcal{B}} \bar{\tau}_{\xi+}^{3} \mathcal{T}_{\mu}\right)\right]+\ldots
\end{aligned}
$$

At one-loop level the matrix elements of the axial current between nucleons of flavor "a" and "b" are 


$$
\begin{aligned}
{ }^{(P Q)} \Gamma_{a b}=\left\langle\left. N_{b}\right|^{(P Q)} j_{\mu, 5} \mid N_{a}\right\rangle= & {\left[\rho_{a b}+\frac{1}{16 \pi^{2} f^{2}}\left(\eta_{a b}-\rho_{a b} \frac{1}{2}\left[w_{a}+w_{b}\right]+y_{j} \eta_{a b}^{(j)}+y_{l} \eta_{a b}^{(l)}\right)\right.} \\
& \left.+c_{a b}+y_{j} c_{a b}^{(j)}+y_{l} c_{a b}^{(l)}\right] 2 \bar{U}_{b} S_{\mu} U_{a}
\end{aligned}
$$

where the wavefunction renormalization contributions are given in eqs. (61) and (63). The $c_{a b}$ 's correspond to contributions from local counterterms involving one insertion of the mass matrix, $m_{Q}$,

$$
\begin{aligned}
{ }^{(P Q)} j_{\mu, 5}^{m_{Q}} & \rightarrow 2\left[b_{1} \overline{\mathcal{B}}^{k j i}\left\{\bar{\tau}_{\xi+}^{3}, \mathcal{M}_{+}\right\}_{i}^{n} S_{\mu} \mathcal{B}_{n j k}\right. \\
& +b_{2}(-)^{\left(\eta_{i}+\eta_{j}\right)\left(\eta_{k}+\eta_{n}\right)} \overline{\mathcal{B}}^{k j i}\left\{\bar{\tau}_{\xi+}^{3}, \mathcal{M}_{+}\right\}_{k}^{n} S_{\mu} \mathcal{B}_{i j n} \\
& +b_{3}(-)^{\eta_{l}\left(\eta_{j}+\eta_{n}\right)} \overline{\mathcal{B}}^{k j i}\left(\bar{\tau}_{\xi+}^{3}\right)_{i}^{l}\left(\mathcal{M}_{+}\right)_{j}^{n} S_{\mu} \mathcal{B}_{l n k} \\
& +b_{4}(-)^{\eta_{l} \eta_{j}+1} \overline{\mathcal{B}}^{k j i}\left(\left(\bar{\tau}_{\xi+}^{3}\right)_{i}^{l}\left(\mathcal{M}_{+}\right)_{j}^{n}+\left(\mathcal{M}_{+}\right)_{i}^{l}\left(\bar{\tau}^{3}\right)_{j}^{n}\right) S_{\mu} \mathcal{B}_{n l k} \\
& +b_{5}(-)^{\eta_{i}\left(\eta_{l}+\eta_{j}\right)} \overline{\mathcal{B}}^{k j i}\left(\bar{\tau}_{\xi+}^{3}\right)_{j}^{l}\left(\mathcal{M}_{+}\right)_{i}^{n} S_{\mu} \mathcal{B}_{n l k}+b_{6} \overline{\mathcal{B}}^{k j i}\left(\bar{\tau}_{\xi+}^{3}\right)_{i}^{l} S_{\mu} \mathcal{B}_{l j k} \operatorname{str}\left(\mathcal{M}_{+}\right) \\
& +b_{7}(-)^{\left(\eta_{i}+\eta_{j}\right)\left(\eta_{k}+\eta_{n}\right)} \overline{\mathcal{B}}^{k j i}\left(\bar{\tau}_{\xi+}^{3}\right)_{k}^{n} S_{\mu} \mathcal{B}_{i j n} \operatorname{str}\left(\mathcal{M}_{+}\right) \\
& \left.+b_{8} \overline{\mathcal{B}}^{k j i} S_{\mu} \mathcal{B}_{i j k} \operatorname{str}\left(\bar{\tau}_{\xi+}^{3} \mathcal{M}_{+}\right)\right]
\end{aligned}
$$

where the coefficients, $b_{i}$, must be determined from data or from lattice calculations.

For the matrix element of $\bar{\tau}^{3}$ between proton states, we find that the diagrams in Fig. 田 give

$$
\begin{aligned}
\rho_{p p}= & g_{A} \\
\eta_{p p}= & g_{A}^{3}\left[L_{u d}-L_{u u}-R_{\eta_{u} \eta_{u}}\right] \\
& +\frac{g_{1} g_{A}^{2}}{3}\left[L_{u d}-2 L_{u u}-L_{j u}-L_{l u}-3 R_{\eta_{u} \eta_{u}}-3 R_{\eta_{u} \eta_{d}}\right] \\
& +\frac{g_{A} g_{1}^{2}}{12}\left[2 L_{u d}-4 L_{u u}-3 L_{j d}+L_{j u}-3 L_{l d}+L_{l u}-3 R_{\eta_{u} \eta_{u}}-6 R_{\eta_{u} \eta_{d}}-3 R_{\eta_{d} \eta_{d}}\right] \\
& +\frac{g_{1}^{3}}{24}\left[3 L_{d d}-L_{u u}+2 L_{u d}-3 L_{j d}+L_{j u}-3 L_{l d}+L_{l u}\right] \\
& -g_{A}\left[L_{u d}-L_{u u}+L_{j u}+L_{l u}\right]-\frac{g_{1}}{2}\left[L_{d d}-L_{u u}+L_{j u}-L_{j d}+L_{l u}-L_{l d}\right] \\
& -\frac{10}{81} g_{\Delta N}^{2} g_{\Delta \Delta}\left[6 J_{u d}+J_{u u}-J_{d d}+2 J_{j d}+2 J_{l d}+\mathcal{T}_{\eta_{u} \eta_{u}}+\mathcal{T}_{\eta_{d} \eta_{d}}-2 \mathcal{T}_{\eta_{u} \eta_{d}}\right] \\
& +\frac{8}{9} g_{\Delta N}^{2} g_{A}\left[K_{u d}+K_{u u}+K_{j u}+K_{l u}+2 \mathcal{S}_{\eta_{u} \eta_{u}}-2 \mathcal{S}_{\eta_{u} \eta_{d}}\right] \\
& -\frac{2}{9} g_{\Delta N}^{2} g_{1}\left[2 K_{d d}-K_{u d}-3 K_{u u}+2 K_{j d}-K_{j u}+2 K_{l d}-K_{l u}+4 \mathcal{S}_{\eta_{d} \eta_{d}}-4 \mathcal{S}_{\eta_{u} \eta_{u}}\right] \\
\eta_{p p}^{(j)}= & g_{A}\left(L_{j u}-L_{u u}\right)+\frac{g_{1}}{2}\left[L_{j d}+L_{j u}-L_{u d}-L_{u u}\right] \\
& -\frac{5}{81} g_{\Delta \Delta} g_{\Delta N}^{2}\left(2 J_{j d}-2 J_{u d}+J_{j u}-J_{u u}\right) \\
& +\frac{1}{3}\left(L_{u u}-L_{j u}\right)\left[\frac{4}{3} g_{A}^{3}+g_{1} g_{A}^{2}+\frac{1}{4} g_{A} g_{1}^{2}\right]+\frac{g_{1}^{3}}{72}\left[5 L_{u u}+9 L_{u d}-5 L_{j u}-9 L_{j d}\right]
\end{aligned}
$$




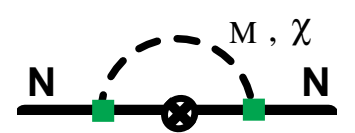

(a)

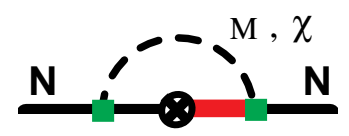

(c)

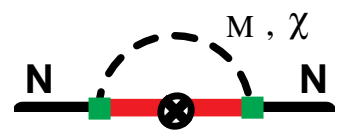

(b)

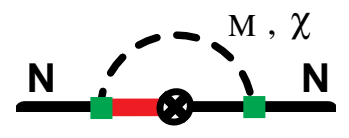

(d)

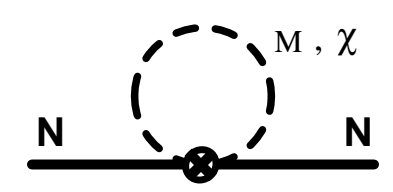

(e)

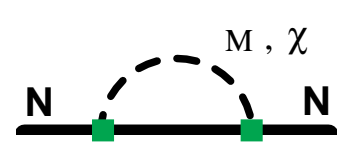

(f)

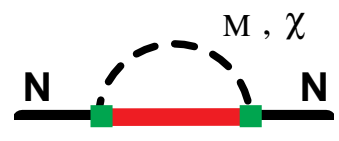

(g)

FIG. 4. One-loop graphs that give contributions of the form $\sim m_{Q} \log m_{Q}$ to the matrix elements of the axial current in the nucleon. A solid, thick-solid and dashed line denote a 70-nucleon, 44-resonance, and a meson, respectively. The solid-squares denote an axial coupling given in eq. (33), while the crossed circle denotes an insertion of the axial current operator. Diagrams (a) to $(e)$ are vertex corrections, while diagrams $(f)$ and $(g)$ give rise to wavefunction renormalization.

$$
\begin{aligned}
& -\frac{2}{9} g_{\Delta N}^{2} g_{1}\left[K_{u u}+2 K_{u d}-K_{j u}-2 K_{j d}\right] \\
\eta_{p p}^{(l)}= & g_{A}\left(L_{l u}-L_{u d}\right)+\frac{g_{1}}{2}\left[L_{l d}+L_{l u}-L_{u d}-L_{d d}\right] \\
& -\frac{5}{81} g_{\Delta \Delta} g_{\Delta N}^{2}\left(2 J_{l d}-2 J_{d d}+J_{l u}-J_{u d}\right) \\
& +\frac{1}{3}\left(L_{u d}-L_{l u}\right)\left[\frac{4}{3} g_{A}^{3}+g_{1} g_{A}^{2}+\frac{1}{4} g_{A} g_{1}^{2}\right]+\frac{g_{1}^{3}}{72}\left[5 L_{u d}+9 L_{d d}-5 L_{l u}-9 L_{l d}\right] \\
& -\frac{2}{9} g_{\Delta N}^{2} g_{1}\left[K_{u d}+2 K_{d d}-K_{l u}-2 K_{l d}\right] \\
c_{p p}= & m_{u}\left(\frac{2}{3} b_{1}+\frac{5}{3} b_{2}-\frac{1}{2} b_{3}+b_{4}+b_{8}\right)+m_{d}\left(-\frac{4}{3} b_{1}-\frac{1}{3} b_{2}+\frac{1}{6} b_{3}-\frac{2}{3} b_{4}+\frac{2}{3} b_{5}-b_{8}\right) \\
& +\left(m_{j}+m_{l}\right)\left(\frac{2}{3} b_{7}-\frac{1}{3} b_{6}\right) \\
c_{p p}^{(j)}= & b_{8}\left(m_{j}-m_{u}\right) \\
c_{p p}^{(l)}= & b_{8}\left(m_{l}-m_{d}\right) \quad,
\end{aligned}
$$

where we have defined the loop function, $K_{a b}=K\left(m_{a b}, \Delta, \mu\right)$ with 


$$
\begin{aligned}
K(m, \Delta, \mu)= & \left(m^{2}-\frac{2}{3} \Delta^{2}\right) \log \left(\frac{m^{2}}{\mu^{2}}\right)+\frac{2}{3} \Delta \sqrt{\Delta^{2}-m^{2}} \log \left(\frac{\Delta-\sqrt{\Delta^{2}-m^{2}+i \epsilon}}{\Delta+\sqrt{\Delta^{2}-m^{2}+i \epsilon}}\right) \\
& +\frac{2}{3} \frac{m^{2}}{\Delta}\left(\pi m-\sqrt{\Delta^{2}-m^{2}} \log \left(\frac{\Delta-\sqrt{\Delta^{2}-m^{2}+i \epsilon}}{\Delta+\sqrt{\Delta^{2}-m^{2}+i \epsilon}}\right)\right)
\end{aligned}
$$

and $\mathcal{S}_{\eta_{a}, \eta_{b}}=\mathcal{H}\left(K_{a a}, K_{b b}, K_{X}\right)$. For the matrix element of $\bar{\tau}^{3}$ between neutron states, we find

$$
\begin{aligned}
& \rho_{n n}=-g_{A} \\
& \eta_{n n}=-g_{A}^{3}\left[L_{u d}-L_{d d}-R_{\eta_{d} \eta_{d}}\right] \\
& +\frac{g_{1} g_{A}^{2}}{3}\left[2 L_{d d}-L_{u d}+L_{j d}+L_{l d}+3 R_{\eta_{u} \eta_{d}}+3 R_{\eta_{d} \eta_{d}}\right] \\
& +\frac{g_{A} g_{1}^{2}}{12}\left[4 L_{d d}-2 L_{u d}-L_{j d}+3 L_{j u}-L_{l d}+3 L_{l u}+3 R_{\eta_{u} \eta_{u}}+6 R_{\eta_{u} \eta_{d}}+3 R_{\eta_{d} \eta_{d}}\right] \\
& +\frac{g_{1}^{3}}{24}\left[L_{d d}-2 L_{u d}-3 L_{u u}-L_{j d}+3 L_{j u}-L_{l d}+3 L_{l u}\right] \\
& +g_{A}\left[L_{u d}-L_{d d}+L_{j d}+L_{l d}\right]+\frac{g_{1}}{2}\left[L_{u u}-L_{d d}+L_{j d}-L_{j u}+L_{l d}-L_{l u}\right] \\
& +\frac{10}{81} g_{\Delta N}^{2} g_{\Delta \Delta}\left[6 J_{u d}+J_{d d}-J_{u u}+2 J_{j u}+2 J_{l u}+\mathcal{T}_{\eta_{u} \eta_{u}}+\mathcal{T}_{\eta_{d} \eta_{d}}-2 \mathcal{T}_{\eta_{u} \eta_{d}}\right] \\
& -\frac{8}{9} g_{\Delta N}^{2} g_{A}\left[K_{u d}+K_{d d}+K_{j d}+K_{l d}+2 \mathcal{S}_{\eta_{d} \eta_{d}}-2 \mathcal{S}_{\eta_{u} \eta_{d}}\right] \\
& -\frac{2}{9} g_{\Delta N}^{2} g_{1}\left[3 K_{d d}+K_{u d}-2 K_{u u}+K_{j d}-2 K_{j u}+K_{l d}-2 K_{l u}+4 \mathcal{S}_{\eta_{d} \eta_{d}}-4 \mathcal{S}_{\eta_{u} \eta_{u}}\right] \\
& \eta_{n n}^{(j)}=g_{A}\left(L_{j d}-L_{u d}\right)+\frac{g_{1}}{2}\left[L_{j d}+L_{j u}-L_{u d}-L_{u u}\right] \\
& -\frac{5}{81} g_{\Delta N}^{2} g_{\Delta \Delta}\left(J_{j d}-J_{u d}+2 J_{j u}-2 J_{u u}\right) \\
& +\frac{1}{3}\left(L_{u d}-L_{j d}\right)\left[\frac{4}{3} g_{A}^{3}+g_{1} g_{A}^{2}+\frac{1}{4} g_{A} g_{1}^{2}\right]+\frac{g_{1}^{3}}{72}\left[5 L_{u d}+9 L_{u u}-5 L_{j d}-9 L_{j u}\right] \\
& -\frac{2}{9} g_{\Delta N}^{2} g_{1}\left[K_{u d}+2 K_{u u}-K_{j d}-2 K_{j u}\right] \\
& \eta_{n n}^{(l)}=g_{A}\left(L_{l d}-L_{d d}\right)+\frac{g_{1}}{2}\left[L_{l d}+L_{l u}-L_{d d}-L_{u d}\right] \\
& -\frac{5}{81} g_{\Delta N}^{2} g_{\Delta \Delta}\left(J_{l d}-J_{d d}+2 J_{l u}-2 J_{u d}\right) \\
& +\frac{1}{3}\left(L_{d d}-L_{l d}\right)\left[\frac{4}{3} g_{A}^{3}+g_{1} g_{A}^{2}+\frac{1}{4} g_{A} g_{1}^{2}\right]+\frac{g_{1}^{3}}{72}\left[5 L_{d d}+9 L_{u d}-5 L_{l d}-9 L_{l u}\right] \\
& -\frac{2}{9} g_{\Delta N}^{2} g_{1}\left[K_{d d}+2 K_{u d}-K_{l d}-2 K_{l u}\right] \\
& c_{n n}=-m_{u}\left(-\frac{4}{3} b_{1}-\frac{1}{3} b_{2}+\frac{1}{6} b_{3}-\frac{2}{3} b_{4}+\frac{2}{3} b_{5}-b_{8}\right)-m_{d}\left(\frac{2}{3} b_{1}+\frac{5}{3} b_{2}-\frac{1}{2} b_{3}+b_{4}+b_{8}\right) \\
& -\left(m_{j}+m_{l}\right)\left(\frac{2}{3} b_{7}-\frac{1}{3} b_{6}\right) \\
& c_{n n}^{(j)}=b_{8}\left(m_{j}-m_{u}\right) \\
& c_{n n}^{(l)}=b_{8}\left(m_{l}-m_{d}\right) \text {. }
\end{aligned}
$$


For the $n p$ matrix element induced by the $\bar{\tau}^{+}$axial current, we find

$$
\begin{aligned}
& \rho_{n p}=g_{A} \\
& \eta_{n p}=-g_{A}^{3} R_{\eta_{u} \eta_{d}} \\
& +\left(\frac{g_{1} g_{A}^{2}}{6}+\frac{g_{1}^{2} g_{A}}{12}\right)\left[2 L_{u d}-2 L_{u u}-2 L_{d d}-L_{j u}-L_{j d}-L_{l u}-L_{l d}\right. \\
& \left.-3 R_{\eta_{u} \eta_{u}}-6 R_{\eta_{u} \eta_{d}}-3 R_{\eta_{d} \eta_{d}}\right] \\
& +\frac{g_{1}^{3}}{24}\left[2 L_{u d}+L_{u u}+L_{d d}-L_{j u}-L_{j d}-L_{l u}-L_{l d}\right]-\frac{g_{A}}{2}\left[L_{j u}+L_{l u}+L_{j d}+L_{l d}\right] \\
& -\frac{10}{81} g_{\Delta N}^{2} g_{\Delta \Delta}\left[4 J_{u d}+J_{u u}+J_{d d}+J_{j u}+J_{l u}+J_{j d}+J_{l d}+2 \mathcal{T}_{\eta_{u} \eta_{u}}+2 \mathcal{T}_{\eta_{d} \eta_{d}}-4 \mathcal{T}_{\eta_{u} \eta_{d}}\right] \\
& +\frac{4}{9} g_{\Delta N}^{2} g_{A}\left[4 K_{u d}+K_{j u}+K_{l u}+K_{j d}+K_{l d}+\mathcal{S}_{\eta_{u} \eta_{u}}+\mathcal{S}_{\eta_{d} \eta_{d}}-2 \mathcal{S}_{\eta_{u} \eta_{d}}\right] \\
& +\frac{1}{9} g_{\Delta N}^{2} g_{1}\left[2 K_{u d}+K_{u u}+K_{d d}-K_{j u}-K_{l u}-K_{j d}-K_{l d}\right] \\
& c_{n p}=\left(m_{u}+m_{d}\right)\left(-\frac{1}{3} b_{1}+\frac{2}{3} b_{2}-\frac{1}{6} b_{3}+\frac{1}{6} b_{4}+\frac{1}{3} b_{5}\right)+\left(m_{j}+m_{l}\right)\left(\frac{2}{3} b_{7}-\frac{1}{3} b_{6}\right),
\end{aligned}
$$

along with $\eta_{n p}^{(j)}=\eta_{n p}^{(l)}=c_{n p}^{(j)}=c_{n p}^{(l)}=0$.

These three independent matrix elements each reduce down to that of QCD when $m_{j} \rightarrow$ $m_{u}$ and $m_{l} \rightarrow m_{d}$. In the isospin limit and taking $\Delta \rightarrow 0$ the axial-vector current matrix elements in QCD, at one-loop in the chiral expansion, are [14,32.

$$
\Gamma_{n p}=g_{A}-\frac{L_{\pi}}{8 \pi^{2} f^{2}}\left(g_{A}\left(1+2 g_{A}^{2}\right)+\frac{2}{9} g_{A} g_{\Delta N}^{2}+\frac{50}{81} g_{\Delta \Delta} g_{\Delta N}^{2}\right),
$$

where we have not shown the contribution from local counterterms involving one insertion of $m_{q}$. We have used the fact that $K_{x}, J_{x} \rightarrow L_{x}$ in the $\Delta \rightarrow 0$ limit.

\section{CONCLUSIONS}

The properties of the neutron and the proton have provided an important benchmark for comparing the predictions of continuum hadronic effective field theories (and models) with nature. They now provide a benchmark for the ever increasing number of lattice QCD calculations, be they quenched, partially-quenched or unquenched. In this paper we have analyzed several observables in two-flavor PQ $\chi \mathrm{PT}$ to allow for extrapolations of current and future lattice simulations of two-flavor QCD. One-loop level computations of the nucleon

masses, magnetic moments, axial-vector currents, as well as the forward matrix elements of isovector twist-2 operators that are directly related to parton distribution functions, have been presented.

\section{ACKNOWLEDGMENTS}

We thank Paul Rakow for useful discussions. This work is supported in part by the U.S. Dept. of Energy under Grants No. DE-FG03-97ER4014. 


\section{REFERENCES}

[1] S.R. Sharpe, Nucl. Phys. B17 (Proc. Suppl.), 146 (1990).

[2] S.R. Sharpe, Phys. Rev. D46, 3146 (1992).

[3] C. Bernard and M.F.L. Golterman, Phys. Rev. D46, 853 (1992).

[4] J.N. Labrenz and S.R. Sharpe, Phys. Rev. D54, 4595 (1996).

[5] M.J. Savage, Nucl. Phys. A700, 359 (2002).

[6] S.R. Sharpe and N. Shoresh, Phys. Rev. D62, 094503 (2000); Nucl. Phys. Proc. Suppl. 83, 968 (2000); M.F.L. Golterman and K.-C. Leung, Phys. Rev. D57, 5703 (1998). S.R. Sharpe, Phys. Rev. D56, 7052 (1997); C.W. Bernard and M.F.L. Golterman, Phys. Rev. D49, 486 (1994).

[7] S.R. Sharpe and N. Shoresh, Int. J. Mod. Phys. A16s1c, 1219 (2001); Phys. Rev. D64, 114510 (2001).

[8] J.-W. Chen and M.J. Savage, hep-lat/0111050.

[9] S.R. Beane and M.J. Savage, hep-lat/0202013.

[10] A.B. Balantekin, I. Bars and F. Iachello, Phys. Rev. Lett. 47, 19 (1981).

[11] A.B. Balantekin and I. Bars, J. Math. Phys. 23, 1239 (1981); J. Math. Phys. 22, 1810 (1981); J. Math. Phys. 22, 1149 (1981).

[12] J.-P. Hurni and B. Morel, J. Math. Phys. 24, 157 (1983).

[13] E. Jenkins and A.V. Manohar, Phys. Lett. B255, 558 (1991).

[14] E. Jenkins and A.V. Manohar, Phys. Lett. B259, 353 (1991).

[15] E. Jenkins, Nucl. Phys. B368, 190 (1992).

[16] E. Jenkins and A.V. Manohar, Baryon Chiral Perturbation Theory, talks presented at the workshop on Effective Field Theories of the Standard Model, Dobogoko, Hungary (1991);

[17] For a recent review see U.-G. Meißner, Essay for the Festschrift in honor of Boris Ioffe, in "Encyclopedia of Analytic QCD", edited by M. Shifman, World Scientific, ISBN-98102-4968-3.

[18] E. Jenkins, Nucl. Phys. B368, 190 (1992); V. Bernard, N. Kaiser and U.-G. Meißner, Z. Phys. C60, 111 (1993).

[19] T. Draper, R.M. Woloshyn and K.F. Liu, Phys. Lett. 234, 121 (1990); D.B. Leinweber, R.M. Woloshyn and T. Draper, Phys. Rev. D43, 1659 (1991); W. Wilcox, T. Draper and K.F. Liu, Phys. Rev. D46, 1109 (1992); S. Capitani et al, hep-lat/9711007; S.J. Dong, K.F. Liu and A.G. Williams, Phys. Rev. D58, 074504 (1998); A.G. Williams, Nucl. Phys. Proc. Suppl. 73, 306 (1999); V. Gadiyak, X.D. Ji and C.W. Jung, Nucl. Phys. Proc. Suppl. 106, 296 (2002).

[20] M.F.L. Golterman and E. Pallante, JHEP 0110, 037 (2001); Nucl. Phys. Proc. Suppl. 106, 335 (2002); hep-lat/0108029.

[21] D. G. Caldi and H. Pagels, Phys. Rev. D10, 3739 (1974).

[22] E. Jenkins, M. Luke, A.V. Manohar and M.J. Savage, Phys. Lett. B302, 482 (1993); B388, 866 (1996)(E).

[23] U.-G. Meißner and S. Steininger Nucl. Phys. B499, 349 (1997).

[24] M. Fukugita, Y. Kuramashi, M. Okawa and A. Ukawa, Phys. Rev. Lett. 75, 2092 (1995); M. Gockeler et al, Phys. Rev. D53, 2317 (1996); M. Gockeler et al Nucl. Phys. Proc. Suppl. 53, 81 (1997); C. Best et al., hep-ph/9706502; R. Horsley [UKQCD Collaboration], Nucl. Phys. Proc. Suppl. 94, 307 (2001); T. Blum, S. Ohta and 
S. Sasaki, Nucl. Phys. Proc. Suppl. 94, 295 (2001); D. Dolgov et al., Nucl. Phys. Proc. Suppl. 94, 303 (2001) S. Sasaki, T. Blum, S. Ohta and K. Orginos [RBC Collaboration], Nucl. Phys. Proc. Suppl. 106, 302 (2002); M. Gockeler, R. Horsley, D. Pleiter, P.E. Rakow and G. Schierholz, hep-ph/0108105; D. Dolgov et al. [LHPC collaboration], hep-lat/0201021.

[25] D. Arndt and M.J. Savage, Nucl.Phys. A697, 429 (2002).

[26] J-.W. Chen and X. Ji, hep-ph/0105197.

[27] J-.W. Chen and X. Ji, Phys. Rev. Lett. 87, 152002 (2001).

[28] W. Detmold, W. Melnitchouk, J.W. Negele, D.B. Renner, and A.W. Thomas, Phys. Rev. Lett. 87, 172001 (2001).

[29] J.-W. Chen and M.J. Savage, nucl-th/0108042.

[30] J-.W. Chen and X. Ji, hep-ph/0105296.

[31] J-.W. Chen and X. Ji, Phys. Rev. Lett. 88, 052003 (2002).

[32] J. Bijnens, H. Sonoda and M.B. Wise, Nucl. Phys. B261, 185 (1985); M.N. Butler, R.P. Springer and M.J. Savage, Nucl. Phys. B399, 69 (1993); M.A. Luty and M.J. White, Phys. Lett. B319, 261 (1993); hep-ph/9304291; R.P. Springer, hep-ph/9508324; M. Kim and S. Kim, Phys. Rev. D58, 074509 (1998); M.J. Savage and J. Walden, Phys. Rev. D55, 5376 (1997); R. Flores-Mendieta, E. Jenkins and A.V. Manohar, Phys. Rev. D58, 094028 (1998); B. Borasoy, Phys. Rev. D59, 054021 (1999); B.R. Holstein, Few. Body. Syst. Suppl. 11,116 (1999); S.-L. Zhu, S. Puglia and M.J. Ramsey-Musolf, Phys. Rev. D63, 034002 (2001); 